\title{
Leptogenesis from oscillations and dark matter
}

\author{
Andrea Caputo $^{\mathrm{a}}$, Pilar Hernandez ${ }^{\mathrm{b}}$, Nuria Rius ${ }^{\mathrm{c}}$ \\ Instituto de Física Corpuscular, Universidad de Valencia and CSIC, Edificio Institutos Investigación, Catedrático Jose Beltrán 2, Paterna 46980, \\ Spain
}

Received: 22 December 2018 / Accepted: 1 July 2019 / Published online: 9 July 2019

(C) The Author(s) 2019

\begin{abstract}
An extension of the Standard Model with Majorana singlet fermions in the $1-100 \mathrm{GeV}$ range can explain the light neutrino masses and give rise to a baryon asymmetry at freeze-in of the heavy states, via their CP-violating oscillations. In this paper we consider extending this scenario to also explain dark matter. We find that a very weakly coupled $B-L$ gauge boson, an invisible QCD axion model, and the singlet majoron model can simultaneously account for dark matter and the baryon asymmetry.
\end{abstract}

\section{Introduction}

The standard model (SM) of particle physics needs to be extended to explain neutrino masses, the missing gravitating matter (DM) and the observed matter-antimatter asymmetry in the universe.

Some of the most minimal extensions of the SM include new fermions, namely two or three sterile Majorana neutrinos (singlets under the full gauge group), which can account for the tiny neutrino masses, through the seesaw mechanism [1-4], and explain the observed matter-antimatter asymmetry through leptogenesis [5]. The simplest version of leptogenesis establishes the source of the matter-antimatter asymmetry in the CP violating the out-of-equilibrium decay of the heavy neutrinos. This scenario requires however relatively large Majorana masses $>10^{8} \mathrm{GeV}[6]$ (or $\sim 10^{6} \mathrm{GeV}$ with flavour effects [7] included), which makes these models difficult to test experimentally. For Majorana neutrinos in the $1-100 \mathrm{GeV}$ range, it has been shown by Akhmedov, Rubakov and Smirnov (ARS) [8] and refined by Asaka and Shaposhnikov (AS) [9] that a different mechanism of leptogenesis is at work. In this case the asymmetries are produced at freezein of the sterile states via their CP-violating oscillations. The

\footnotetext{
a e-mail: andrea.caputo@uv.es

be-mail: pilar.hernandez@cern.ch

c e-mail: nuria.rius@ific.uv.es
}

original ARS proposal did not include flavour effects and needs at least three Majorana species, while AS have shown that flavour effects can make it work with just two species. In the rest of the paper we will refer indistinctively to both scenarios as baryogenesis from oscillations (BO). In both cases, the lepton asymmetry is reprocessed into a baryonic one by electroweak sphalerons [10]. The extra heavy neutrinos in this case could be produced and searched for in beam dump experiments and colliders (see [11-23] for an incomplete list of works), possibly giving rise to spectacular signals such as displaced vertices $[15,16,18,20,21]$. Since only two sterile neutrinos are needed to generate the baryon asymmetry [9,24-39], the lightest sterile neutrino in the $\mathrm{keV}$ range can be very weakly coupled and play the role of DM [40]. This is the famous $v$ MSM [9]. However the stringent X-ray bounds imply that this scenario can only work in the presence of a leptonic asymmetry [41] significantly larger than the baryonic one, which is quite difficult to achieve. A recent update of astrophysical bounds on this scenario can be found in $[42,43]$.

In this paper our main goal is to consider scenarios compatible with Majorana masses in the $1-100 \mathrm{GeV}$ range and study the conditions under which the models can explain DM without spoiling ARS leptogenesis. ${ }^{1}$ In particular, we will focus on models that are minimal extensions of the type I seesaw model with three singlet neutrinos. We will first consider an extension involving a gauged $B-L$ model [45], which includes an extra gauge boson and can explain DM in the form of a non-thermal $\mathrm{keV}$ neutrino. We will then consider an extension which includes a CP axion [46] that can solve the strong CP problem and explain DM in the form of cold axions. Finally we consider the majoron singlet model $[47,48]$ which can also explain DM under certain conditions both in the form of a heavy majorana neutrino or a majoron.

\footnotetext{
1 Some very recent work along these lines in the scotogenic model can be found in [44].
} 
The plan of the paper is as follows. We start by briefly reviewing the ARS mechanism and the essential ingredients and conditions that need to be met when the sterile neutrinos have new interactions. In Sect. 3 we discuss the gauged $B-L$ model, in Sect. 4, we study the invisible axion model with sterile neutrinos and in Sect. 5 we reconsider the singlet majoron model. In Sect. 6 we conclude.

\section{Leptogenesis from oscillations}

For a recent extensive review of the ARS mechanism see [49]. The model is just the type I seesaw model with three neutrino singlets, $N_{i}, i=1-3$, which interact with the SM only through their Yukawa couplings. The Lagrangian in the Majorana mass basis is

$$
\begin{aligned}
\mathcal{L}= & \mathcal{L}_{\mathrm{SM}}+i \bar{N}_{i} \gamma^{\mu} \partial_{\mu} N_{i} \\
& -\left(Y_{\alpha i} \bar{L}_{\alpha} N_{i} \Phi+\frac{m_{N_{i}}}{2} \bar{N}_{i}^{c} N_{i}+\text { h.c. }\right) .
\end{aligned}
$$

In the early Universe before the electroweak (EW) phase transition, the singlet neutrinos are produced through their Yukawa couplings in flavour states, which are linear combinations of the mass eigenstates. Singlet neutrinos then oscillate, and since $\mathrm{CP}$ is not conserved, lepton number $L$ gets unevenly distributed between different flavours. At high enough temperatures $T \gg m_{N_{i}}$, total lepton number vanishes, in spite of which a surplus of baryons over antibaryons can be produced, because the flavoured lepton asymmetries are stored in the different species and transferred at different rates to the baryons. As long as full equilibration of the sterile states is not reached before the EW phase transition $\left(T_{\mathrm{EW}} \sim 140 \mathrm{GeV}\right)$, when sphaleron processes freeze-out, a net baryon asymmetry survives. It is essential that at least one of the sterile neutrinos does not equilibrate by $t_{\mathrm{EW}}$. The rate of interactions of these neutrinos at temperatures much higher than their mass can be estimated to be

$\Gamma_{\alpha} \propto \kappa y_{\alpha}^{2} T$,

where $y_{\alpha}$ are the eigenvalues of the neutrino Yukawa matrix, $T$ is the temperature and $\kappa=$ few $10^{-3}$ [50-52]. The Hubble expansion rate in the radiation dominated era is

$H(T)=\sqrt{\frac{4 \pi^{3} G_{N} g_{*}}{45}} T^{2} \equiv \frac{T^{2}}{M_{P}^{*}}$.

where $g_{*}$ is the number of relativistic degrees of freedom $\left(g_{*} \sim 100\right.$ above the EW phase transition). The requirement that no equilibration is reached before $t_{\mathrm{EW}}$ is:

$\Gamma_{\alpha}\left(T_{\mathrm{EW}}\right) \leq H\left(T_{\mathrm{EW}}\right)$,

which implies yukawa couplings of order

$y_{\alpha} \lesssim 10^{-7}$, i.e. not much smaller than the electron yukawa. These yukawa couplings are compatible with the light neutrino masses for Majorana masses in the $1-100 \mathrm{GeV}$ range.

Any model that extends the one described above with new fields/interactions should be such that the new interactions do not increase the equilibration rate of the sterile neutrinos for the out-of-equilibrium requirement in the ARS mechanism to be met. We will now consider the implications of this requirement on various extensions of the minimal seesaw model of Eq. (1) that are well motivated by trying to explain also the dark matter and in one case also the strong $\mathrm{CP}$ problem.

\section{B-L gauge symmetry}

The SM is invariant under an accidental global $U(1)_{B-L}$ symmetry, that couples to baryon minus lepton number. If one promotes this symmetry to a local one [45], the model needs to be extended with three additional right handed neutrinos to avoid anomalies, which interestingly makes the type I seesaw model the minimal particle content compatible with this gauge symmetry. In this case, we have interactions between SM lepton and quark fields with the new gauge boson, $V_{\mu}$, as well as an additional term involving sterile neutrinos

$$
\mathcal{L} \supset g_{B-L}\left(\sum_{f} Q_{B-L}^{f} V_{\mu} \bar{f} \gamma_{\mu} f-\sum_{a} V_{\mu} \bar{N}_{a} \gamma^{\mu} N_{a}\right),
$$

where $Q_{B-L}^{f}=1 / 3,-1$ for quarks and leptons respectively. We also assume the presence of a scalar field $\phi$, with charge $B-L$ charge 2:

$\mathcal{L} \supset\left(D_{\mu} \phi\right)^{\dagger} D_{\mu} \phi-V(\phi)-\frac{h_{N}}{2} \bar{N}^{c} N \phi+h . c .$,

that gets an expectation value $\langle\phi\rangle$, breaking $B-L$ spontaneously, ${ }^{2}$ and giving a mass to both the gauge boson and the sterile neutrinos:

$m_{V}=2 \sqrt{2} g_{B-L}\langle\phi\rangle, \quad m_{N_{i}}=h_{N_{i}}\langle\phi\rangle$.

A massive higgs from the $B-L$ breaking, $\sigma$, remains in the spectrum with a mass that we can assume to be $M_{\sigma} \sim\langle\phi\rangle$.

Existing constraints on this model come from direct searches for $V$ in elastic neutrino-electron scattering, $V$ gauge boson production at colliders, Drell-Yan processes and new flavour changing meson decays [53-60]. The status of these searches is summarized in Fig. 1, adapted from [61] (see also [62-64]). For masses, $1 \mathrm{GeV} \leq m_{V} \leq 10 \mathrm{GeV}$, $g_{B-L}$ is bounded to be smaller than $\sim 10^{-4}$, while the limit is weaker for larger masses. The improved prospects to search

\footnotetext{
2 The Stuckelberg mechanism cannot be used here, because we need heavy neutrinos.
} 


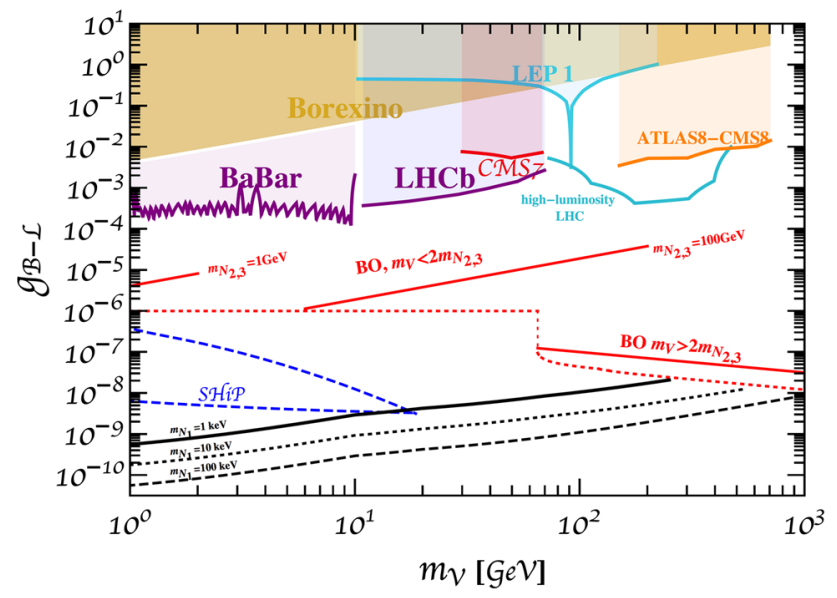

Fig. 1 Summary of present (shaded regions) and future (unshaded) constraints on $g_{B-L}$ and $m_{V}$ for the $B-L$ model, adapted from Ref. [61]. The dashed region labeled SHIP is the sensitivity of bremsstrahlung searches in SHIP [66,67]. The solid, dotted and dashed black lines correspond to the correct DM relic abundance in the form of sterile neutrinos of mass 1,10 and $100 \mathrm{keV}$ respectively. The dotted red line indicates the lower limit on the gauge coupling for which $V$ is in thermal equilibrium. The solid red lines correspond to the upper bounds for successful BO leptogenesis for $m_{N_{2,3}}=1$ and $100 \mathrm{GeV}$

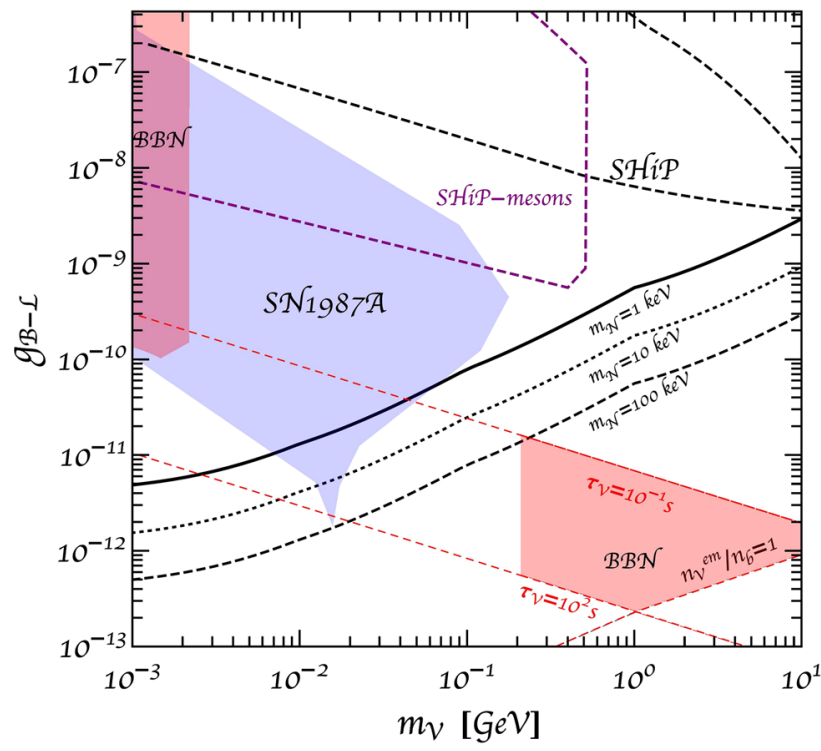

Fig. 2 Shaded regions are presently excluded by supernova observations [68], BBN bounds estimated in [69] and the combined beam dump experiments from [70]. Unshaded regions represent the reach of SHIP from meson decay and bremsstrahlung searches [66]. The curves indicate the values of $\left(g_{B-L}, m_{V}\right)$ where the lightest sterile neutrino $N_{1}$ can account for the whole dark matter for three values of the neutrino masses, $m_{N}=1,10,100 \mathrm{keV}$ in solid, dotted and dashed lines

for right-handed neutrinos exploiting the $U(1)_{B-L}$ interaction have been recently studied in [61], where the authors consider the displaced decay of the $N$ at the LHC and the proposed SHIP beam dump experiment [65].
For $m_{V} \leq 1 \mathrm{GeV}$ the strongest constraints come from supernova cooling [68,71-73], beam dump searches [70,7478] and big bang nucleosynthesis (BBN) [69,70,79-81]. Recent updates on these bounds are compiled in Fig. 2. The lower mass region labeled BBN is excluded by the effect of $\Delta N_{\text {eff }}$ on the expansion, while the higher mass BBN region is excluded because the injection of electromagnetic energy from the $V$ decay to charged particles during nucleosynthesis distorts the abundance of light elements. These BBN constraints have been evaluated in detail in Refs. $[79,80]$. In the relevant region of parameter space, they are seen to depend on the lifetime of the decaying particle and its abundance per baryon prior to decay. The corresponding region in Fig. 2 is a sketch of the excluded region in the latter analysis, which is approximately bounded by the lines corresponding to the lifetimes $\tau_{V} \in[0.1-100] \mathrm{s}$, the threshold for $V$ hadronic decays, $m_{V} \geq 2 m_{\pi}$, and the line corresponding to the fraction of the $V$ decaying to charged particles per baryon, $n_{V}^{e m} / n_{b}=1$.

At least two of the neutrinos will be involved in the BO mechanism and their masses must be in the $1-100 \mathrm{GeV}$ range. We need to ensure that the new $B-L$ interactions do not bring them to thermal equilibrium before $t_{E W}$, which will set an upper bound on $g_{B-L}$. It it important to know however if the $B-L$ gauge boson is in thermal equilibrium which also depends on $g_{B-L}$. For $m_{V} \lesssim 65 \mathrm{GeV}$, the dominant process is the scattering $t t \leftrightarrow V H$ with a rate that can be estimated to be

$\Gamma(t t \rightarrow V H) \simeq \frac{\zeta(3) g_{B-L}^{2} y_{t}^{2}}{144 \pi^{3}} T \log \left(\frac{T}{m_{t}(T)}\right)$,

where $y_{t}$ is the Yukawa of the top quark and $m_{t}(T)$ is its thermal mass. This rate is larger than the Hubble expansion somewhere above the EW transition provided $g_{B-L} \gtrsim 10^{-6}$. For larger masses of the gauge boson $\left(m_{V} \gtrsim 65 \mathrm{GeV}\right)$ the process $V \leftrightarrow f \bar{f}$ kinematically opens up at high temperature and one has to consider the decay and inverse decay with a rate

$$
\begin{aligned}
\Gamma(V \leftrightarrow f \bar{f})= & \sum_{f} \frac{g_{B-L}^{2} N_{C} Q_{f}^{2} m_{V}}{12 \pi}\left(1+\frac{2 m_{f}^{2}}{m_{V}^{2}}\right) \\
& \times\left(1-\frac{4 m_{f}^{2}}{m_{V}^{2}}\right)^{1 / 2},
\end{aligned}
$$

where $N_{C}=3(1)$ and $Q_{f}=1 / 3(-1)$ for quarks(leptons). The sum is over all the standard model fermions whose thermal mass is such that $m_{V}(T) \geq 2 m_{f}(T)$. The lower limit on $g_{B-L}$ for the thermalization of the $B-L$ boson is shown as a dotted red line in Fig.1. Provided the $B-L$ boson is in thermal equilibrium we have to consider its interactions with the sterile neutrinos driving leptogenesis. Assuming that the $V$ boson is lighter than $2 m_{N}$, so that the decay $V \rightarrow N N$ is kinematically forbidden, the dominant contribution [82] comes from the scattering processes with the fermions and 

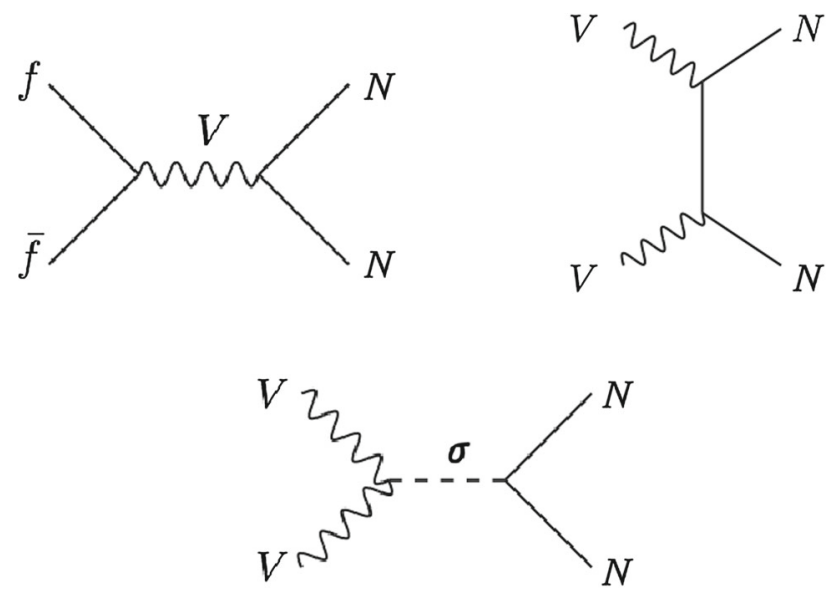

Fig. 3 Production of sterile neutrinos via the new gauge interaction

gauge bosons in Fig. 3. To ensure these processes do not equilibrate the sterile neutrinos, we should have

$\langle\Gamma(V V \rightarrow N N)\rangle_{T=\langle\phi\rangle}<H(T=\langle\phi\rangle)$

Assuming $M_{\sigma} \sim\langle\phi\rangle \gg m_{V}, m_{N}$,

$\langle\Gamma(V V \rightarrow N N)\rangle \sim \frac{g_{B-L}^{4} m_{N}^{2}}{m_{V}^{4}} \frac{3 \zeta(3)}{4 \pi^{3}} T^{3}$,

and using Eq. (8) we get

$g_{B-L}<5 \cdot 10^{-6}\left(\frac{m_{V}}{1 \mathrm{GeV}}\right)\left(\frac{1 \mathrm{GeV}}{m_{N}}\right)^{2 / 3}$.

This upper bound is shown by the red solid line in Fig. 1. In the next section we will include these new interactions in the equations for the generation of the baryon asymmetry, and our results confirm the naive estimate in Eq. (13).

Finally we consider the production of neutrinos through the decay of $\sigma \rightarrow N N$, which is relevant for $T \geq M_{\sigma}$, since $M_{\sigma} \gg m_{N}$. The requirement that this process does not thermalize the sterile neutrinos implies that the decay rate, $\Gamma_{\sigma}$, is slower than the Hubble rate at $T \geq M_{\sigma}$ :

$\Gamma_{D}\left(M_{\sigma}\right)=\frac{h_{N}^{2} M_{\sigma}}{16 \pi} \lesssim H\left(M_{\sigma}\right)$.

For $m_{N}=1-100 \mathrm{GeV}$, using Eq. (8), we get

$M_{\sigma} \sim\langle\phi\rangle \geq 2 \times 10^{5}-5 \times 10^{6} \mathrm{GeV}$.

On the other hand, for $m_{V} \geq 2 m_{N}$ the dominant production goes via the decay of the gauge boson into two sterile neutrinos $V \rightarrow N N$, which, if kinematically allowed, scales with $g_{B-L}^{2}$. In this case the decay rate is

$\Gamma(V \rightarrow N N)=\frac{g_{B-L}^{2} m_{V}}{24 \pi}\left(1-\frac{4 m_{N}^{2}}{m_{V}^{2}}\right)^{3 / 2}$.

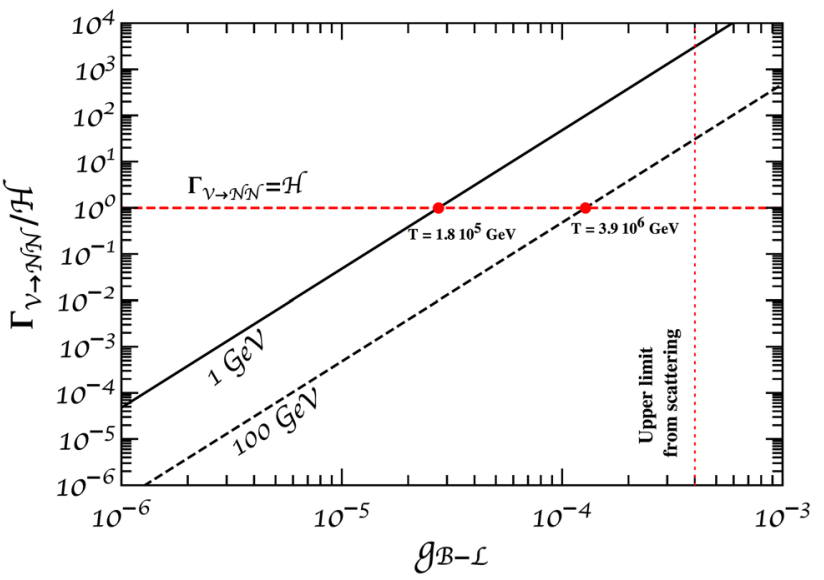

Fig. $4 \Gamma(T) / H(T)$ including thermal effects for $m_{V}$ and two values of the heavy neutrino masses $m_{N}=1,100 \mathrm{GeV}$ for $T=2 \times$ the threshold temperature

Requiring that it is smaller than $H\left(T_{E W}\right)$ implies for $m_{N} \ll$ $m_{V}$

$g_{B-L} \lesssim 10^{-7}\left(\frac{100 \mathrm{GeV}}{m_{V}}\right)^{\frac{1}{2}}$.

One may worry if thermal mass corrections can allow the decay $V \rightarrow N N$ at large temperatures even if $m_{V} \leq 2 m_{N}$. At high enough temperatures both sterile neutrinos and the gauge boson acquire thermal corrections to the masses of the form

$m(T) \sim g_{B-L} T$.

The thermal mass of the gauge boson is larger than that of the sterile neutrino, because all fermions charged under $B-L$ will contribute to the former and only the gauge boson loop contributes to the later [83]:

$m_{V}^{T}=m_{V}+\sqrt{\frac{4}{3}} g_{B-L} T, \quad m_{N}^{T}=m_{N}+\frac{1}{\sqrt{8}} g_{B-L} T$.

We substitute the temperature dependent mass in Eq. (16) and we show in Fig. 4 the ratio $\Gamma(V \rightarrow N N) / H$ close to the minimum threshold temperature (where $m_{V}^{T} \geq 2 m_{N}^{T}$ ), for $m_{N}=1$ and $100 \mathrm{GeV}$ as a function of $g_{B-L}$. The upper limit for $g_{B-L}$ are less stringent than those derived from $2 \rightarrow 2$ processes in Eq. (13). We now evaluate in detail the effect on BO induced by the new scatterings of Fig. 3. Leptogenesis in the presence of a new $B-L$ gauge interaction has been recently studied in [82], although not in the context of BO, which as far as we know has not been considered before.

\subsection{Leptogenesis}

The sterile neutrinos relevant for leptogenesis are the heavier ones, $m_{N_{2,3}}$, with masses in the $1-100 \mathrm{GeV}$, and we focus on the scenario where $m_{V} \leq 2 m_{N_{2,3}}$. We now explain how to 
include the terms involving the $B-L$ gauge interactions in the quantum kinetic equations for BO leptogenesis as derived in [31]. Following Raffelt-Sigl approach [84], we consider a density matrix, $\rho(k)$, describing the expectation value of number densities of $N$ and a density matrix describing the corresponding anti-particles, $\bar{\rho}(k) .{ }^{3}$ These equations are complemented with three equations involving the slow varying chemical potentials, $\mu_{B / 3-L_{\alpha}}$. The modification of the kinetic equations induced by the $B-L$ interactions is the addition of new collision terms in the equations for $\rho$ and $\bar{\rho}$, that have the same flavour structure as the neutral current contribution considered in [84]. As explained above the most relevant contributions come from the scattering processes: $\bar{f} f \leftrightarrow N \bar{N}$ and $V V \leftrightarrow N \bar{N}$. The latter is enhanced at high temperatures, but of course will only be relevant if the $V^{\prime} s$ are in thermal equilibrium, which we assume in the following.

The additional collision terms, from the first process, in the equation for the evolution $\rho(k)$ can be writen in the form:

$$
\begin{aligned}
(\dot{\rho}(k))_{B-L}^{f f \rightarrow N N}= & \frac{1}{2} \int \prod_{i} \frac{d^{3} p_{i}}{(2 \pi)^{3} 2 E_{i}}(2 \pi)^{4} \delta\left(p_{1}+p_{2}-p_{3}-k\right) \\
& \times \sum_{\text {spins }}\left|\mathcal{M}\left(\bar{f}\left(p_{1}\right) f\left(p_{2}\right) \rightarrow \bar{N}\left(p_{3}\right) N(k)\right)\right|^{2} \\
& \times f_{1}^{e q} f_{2}^{e q}\left[2-\{r, \bar{r}\}-f_{4}^{e q}\{r, 1-\bar{r}\}-f_{3}^{e q}\{\bar{r}, 1-r\}\right],
\end{aligned}
$$

where $f_{i}^{e q} \equiv f_{F}\left(p_{i}\right)$ is the Fermi-Dirac equilibrium distribution function of the particle with momentum $p_{i}$ with $p_{4} \equiv k ;\{$,$\} is the anticommutator, and the normalized matri-$ ces are:

$r(k) \equiv \frac{\rho(k)}{f_{F}(k)}, \quad \bar{r}\left(p_{3}\right) \equiv \frac{\bar{\rho}\left(p_{3}\right)}{f_{F}\left(p_{3}\right)}$.

The additional collision terms for $\bar{\rho}$ have the same form with the substitution $k \leftrightarrow p_{3}$.

For the second process we have similarly:

$$
\begin{aligned}
(\dot{\rho}(k))_{B-L}^{V V \rightarrow N N}= & \frac{1}{2} \int \prod_{i} \frac{d^{3} p_{i}}{(2 \pi)^{3} 2 E_{i}}(2 \pi)^{4} \delta\left(p_{1}+p_{2}-p_{3}-k\right) \\
& \times \sum_{\text {spins }}\left|\mathcal{M}\left(V\left(p_{1}\right) V\left(p_{2}\right) \rightarrow \bar{N}\left(p_{3}\right) N(k)\right)\right|^{2} \\
& \times f_{1}^{e q} f_{2}^{e q}\left[2-\{r, \bar{r}\}-f_{4}^{e q}\{r, 1-\bar{r}\}-f_{3}^{e q}\{\bar{r}, 1-r\}\right],
\end{aligned}
$$

where $f_{i}^{e q}$ is the equilibrium distribution function of the particle with momentum $p_{i}$, ie. Bose-Einstein, $f_{i}^{e q} \equiv f_{B}\left(p_{i}\right)$, for $i=1,2$ and Fermi-Dirac, $f_{i}^{e q} \equiv f_{F}\left(p_{i}\right)$, for $i=3$, 4 .

\footnotetext{
${ }^{3}$ We can neglect Majorana masses in the range of masses considered and therefore particles and antiparticles correspond to the two helicity states.
}

As usual we are interested in the evolution in an expanding universe, where the density matrices depend on momentum, $y \equiv p / T$ and the scale factor or inverse temperature $x \propto$ $T^{-1}$. We consider the averaged momentum approximation, which assumes that all the momentum dependence factorizes in the Fermi-Dirac distribution and the density $r$ is just a function of the scale factor, ie. $\rho(x, y)=f_{F}(y) r(x)$. In this approximation we can do the integration over momentum and the $B-L$ terms in the equation for $r$ and $\bar{r}$ become:

$$
\begin{aligned}
\left(x H \frac{d r}{d x}\right)_{B-L}= & \left(x H \frac{d \bar{r}}{d x}\right)_{B-L}=\frac{\left\langle\gamma_{V}^{(0)}\right\rangle}{2}(2-\{r, \bar{r}\}) \\
& -\left\langle\gamma_{V}^{(1)}\right\rangle(r+\bar{r}-\{r, \bar{r}\}),
\end{aligned}
$$

where $H$ is the Hubble expansion parameter.

The averaged rates including the two processes in Eqs. (20) and (22) (assuming in the latter the $Z^{\prime} s$ are in equilibrium) are computed in the appendix with the result:

$$
\begin{aligned}
& \left\langle\gamma_{V}^{(0)}\right\rangle=\left(3.2(3) \times 10^{-3}+2.95 \times 10^{-4} \frac{m_{N}^{2} T^{2}}{m_{V}^{4}}\right) g_{B-L}^{4} T, \\
& \left\langle\gamma_{V}^{(1)}\right\rangle=\left(3.4(1) \times 10^{-4}+3.55 \times 10^{-5} \frac{m_{N}^{2} T^{2}}{m_{V}^{4}}\right) g_{B-L}^{4} T,
\end{aligned}
$$

where the two terms inside the brackets correspond respectively to the $f \bar{f}$ and $V V$ channels, and are valid for $T<$ $T_{\max } \equiv M_{\sigma} \sim\langle\phi\rangle=\frac{m_{V}}{2 \sqrt{2} g_{B-L}}$. Note the different temperature dependence of the two contributions. The growth of the $V V \leftrightarrow N N$ at high temperatures originates in the contribution of the longitudinal polarization of the $\mathrm{V}$ bosons when the temperature is below the scalar mass, $M_{\sigma}$. For higher temperature, $T \geq T_{\max }$, the contribution of the physical scalar $\sigma$ has to be included, leading to a rate $\propto T$. The new interactions do not modify the chemical potential dependent terms, nor the evolution equation for $\mu_{B / 3-L_{\alpha}}$. The equations are therefore those in [31] with the additional $B-L$ terms in Eq. (23).

To illustrate the effect of the $B-L$ gauge interaction, we have considered the test point of Ref. [31] with masses for the heavy steriles $m_{N_{2,3}} \sim 0.8 \mathrm{GeV}$. Within the parameter space of successful leptogenesis, this point was chosen because it leads to charmed meson decays to heavy sterile neutrinos that could be observable in SHIP, and furthermore this measurement, in combination with input from neutrinoless double beta decay and $\mathrm{CP}$ violation in neutrino oscillations, could provide a quantitative prediction of the baryon asymmetry. Adding the $B-L$ terms to the equations for $r$ and $\bar{r}$ of [31], and solving them numerically (for details on the method see [31]) we obtain the curves in Fig. 5. The rates depend on $m_{V}$ so we choose $m_{V}=1 \mathrm{GeV}$. The evolution of 


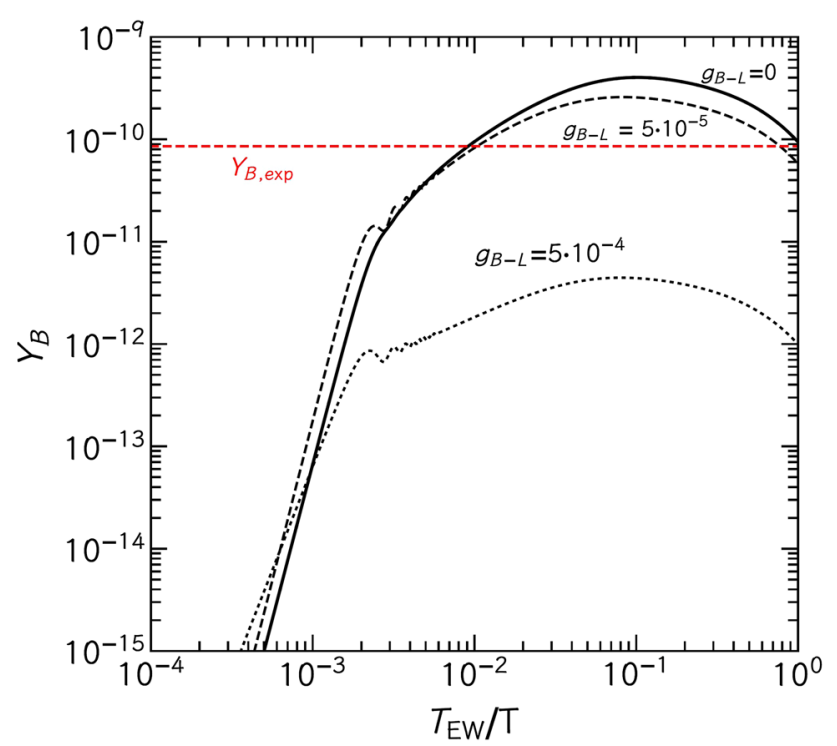

Fig. $5 Y_{B}$ as a function of $T_{\mathrm{EW}} / T$ for the $Y$ and $m_{N}$ parameters corresponding to the test point in [31] and $g_{B-L}=0$ (solid), $g_{B-L}=$ $5 \times 10^{-5}$ (dashed), $g_{B-L}=5 \times 10^{-4}$ (dotted). The horizontal line is the observed value

the baryon asymmetry as a function of $T_{\mathrm{EW}} / T$ is shown by the solid line of Fig. 5 in the absence of $B-L$ interactions or for a sufficiently small value of $g_{B-L}$. The suppression of the asymmetry is visible for larger values of $g_{B-L} \gtrsim$ few $\times 10^{-5}$ as shown by the dashed and dashed-dotted lines. The naive expectations in Fig. 1 is therefore confirmed and we do not expect a significant modification of the baryon asymmetry of the minimal model, as long as $g_{B-L}$ satisfies the bound in Eq. (13).

\subsection{Dark matter}

Now we want to discuss possible dark matter candidates in the $B-L$ scenario without spoiling the $\mathrm{BO}$ mechanism, which as we have seen imposes a stringent upper bound on the gauge coupling, $g_{B-L}$. We will be interested in the region where the $V$ boson can decay to the lightest neutrino, ie $m_{V} \geq 2 m_{N_{1}}$. The small value needed for $g_{B-L}$ suggests to consider the possibility of a freeze-in scenario $[85,86]$, where the gauge boson does not reach thermalization, and neither does the lightest sterile neutrino, $N_{1}$. The status of dark matter in a higher mass range through freeze-out has been recently updated in [64].

As it is well known, $N_{1}$ in the $\mathrm{keV}$ mass range is sufficiently long lived to provide a viable warm DM candidate $[40,41]$. The $B-L$ model is as we will see a simple extension of the vMSM [9], which avoids the need of huge lepton asymmetries to evade $\mathrm{X}$-ray bounds. In our scenario the $\mathrm{keV}$ state is produced from the decay $V \rightarrow N_{1} N_{1}$, while the lifetime of $N_{1}$, relevant in X-ray bounds, is controlled also by mixing, which can be sufficiently small, in a technically natural way, as in the vMSM scenario (provided the lightest neutrino mass is small enough). A similar scenario for DM has been studied in [67]. We now quantify the parameter space for successful DM and leptogenesis in this scenario.

We assume that the abundance of $V$ and $N_{1}$ is zero at a temperature below the EW phase transition where all the remaining particles in the model are in thermal equilibrium. All fermions in the model couple to the $V$ and therefore its production is dominated by the inverse decay process: $f \bar{f} \rightarrow V$. The kinetic equation describing the production of $V$ is the following:

$$
\begin{aligned}
\dot{n}_{V}+3 H n_{V}= & \sum_{f} \int \frac{d^{3} p_{f}}{(2 \pi)^{3} 2 E_{f}} \frac{d^{3} p_{\bar{f}}}{(2 \pi)^{3} 2 E_{\bar{f}}} \frac{d^{3} p_{V}}{(2 \pi)^{3} 2 E_{V}} \\
& \times(2 \pi)^{4} \delta^{4}\left(p_{V}-p_{f}-p_{\bar{f}}\right)\left[|M|_{f \bar{f} \rightarrow V}^{2} f_{f} f_{\bar{f}}\left(1+f_{V}\right)+\right. \\
& \left.-|M|_{V \rightarrow f \bar{f}}^{2} f_{V}\left(1-f_{f}\right)\left(1-f_{\bar{f}}\right)\right],
\end{aligned}
$$

where $f_{i}(p)$ are the distribution function of the particle, $i$, with momentum $p$, and

$n_{i}=g_{i} \int \frac{d^{3} p}{(2 \pi)^{3}} f_{i}(p)$,

is the number density, with $g_{i}$ the number of spin degrees of freedom. $g_{f}=4$ for a Dirac fermion, $g_{N}=2$ for a Majorana fermion and $g_{V}=3$ for a massive gauge boson. $M$ is the amplitude for the decay $V \rightarrow f \bar{f}$ at tree level.

The sum over $f$ is over all fermions, but we can safely neglect the contribution of the $N_{1}$ and also those that are nonrelativistic. We can also neglect the Pauli-blocking and stimulated emission effects $\left(f_{i} \pm 1 \sim \pm 1\right)$ and approximate the distribution function in equilibrium for fermions and bosons by the Maxwell-Boltzmann, $f_{i}\left(p_{i}\right)=f^{e q}\left(p_{i}\right)=e^{-E_{i} / T}$. Taking into account the relation

$|M|_{V \rightarrow f \bar{f}}^{2}=|M|_{f \bar{f} \rightarrow V}^{2}$,

and the principle of detailed balance

$f_{f}^{e q} f_{\bar{f}}^{e q}=f_{V}^{e q}$,

the equation can be simplified to

$$
\begin{gathered}
\dot{n}_{V}+3 H n_{V}=-\sum_{f} \int \frac{d^{3} p_{f}}{(2 \pi)^{3} 2 E_{f}} \frac{d^{3} p_{\bar{f}}}{(2 \pi)^{3} 2 E_{\bar{f}}} \frac{d^{3} p_{V}}{(2 \pi)^{3} 2 E_{V}} \\
(2 \pi)^{4} \delta^{4}\left(p_{V}-p_{f}-p_{\bar{f}}\right)|M|_{V \rightarrow f \bar{f}}^{2}\left(f_{V}\left(p_{V}\right)-f^{e q}\left(p_{V}\right)\right) .
\end{gathered}
$$

As long as $f_{V} \ll f^{e q}$, the first term on the right-hand side can be neglected and the equation simplifies further to:

$\dot{n}_{V}+3 H n_{V} \simeq 3 \sum_{f} \frac{m_{V}^{2} \Gamma_{V \rightarrow f \bar{f}}}{2 \pi^{2}} T K_{1}\left(\frac{m_{V}}{T}\right)$, 
where $K_{1}$ is the first modified Bessel Function of the 2nd kind. The decay width in the $V$ rest frame is given by

$$
\begin{aligned}
\Gamma(V \rightarrow f \bar{f})= & \frac{g_{B-L}^{2} N_{C} Q_{f}^{2} m_{V}}{12 \pi}\left(1+\frac{2 m_{f}^{2}}{m_{V}^{2}}\right) \\
& \times\left(1-\frac{4 m_{f}^{2}}{m_{V}^{2}}\right)^{1 / 2},
\end{aligned}
$$

where $N_{C}=3(1)$ and $Q_{f}=1 / 3(-1)$ for quarks(leptons).

As usual we define the yield of particle $i$ as

$Y_{i}=\frac{n_{i}}{s}$,

where $s$ is the entropy density

$s=\frac{2 \pi^{2}}{45} g_{s}^{*} T^{3}$,

and we can assume $g_{s}^{*} \simeq g^{*}$. We also consider the averaged momentum approximation which amounts to assuming that $f_{V}$ has the same momentum dependence as $f^{e q}$. Changing variable from time to temperature, the final evolution equation for $Y_{V}$ reads:

$$
\begin{aligned}
\frac{d Y_{V}}{d T}= & -3 \sum_{f} \frac{m_{V}^{2} \Gamma_{V \rightarrow f \bar{f}}}{2 \pi^{2} H s\left[1+\frac{1}{3} \frac{T g_{*}}{g_{*} d T}\right]} K_{1}\left(\frac{m_{V}}{T}\right) \\
& \times\left(1-\frac{s Y_{V}}{n_{V}^{e q}}\right),
\end{aligned}
$$

where $n_{V}^{e q}=\frac{3}{2 \pi^{2}} m_{V}^{2} T K_{2}\left(m_{V} / T\right)$.

The production of $N_{1}$ is dominated by the decay $V \rightarrow$ $N_{1} N_{1}$. There is also the contribution via mixing with the active neutrinos but this is negligible for mixings that evade present $\mathrm{X}$ ray bounds. Neglecting the inverse processes, the evolution equation for $n_{1}$ is

$\dot{n}_{1}+3 H n_{1}=2 \frac{K_{1}(x)}{K_{2}(x)} \Gamma\left(V \rightarrow N_{1} N_{1}\right) n_{V}$,

and in terms of the yield

$$
\frac{d Y_{N_{1}}}{d T}=-\frac{2}{H T\left[1+\frac{1}{3} \frac{T d g_{*}}{g_{*} d T}\right]} \frac{K_{1}(x)}{K_{2}(x)} \Gamma\left(V \rightarrow N_{1} N_{1}\right) Y_{V},
$$

where

$\Gamma\left(V \rightarrow N_{1} N_{1}\right)=\frac{g_{B-L}^{2} m_{V}}{24 \pi}\left(1-\frac{4 m_{N_{1}}^{2}}{m_{V}^{2}}\right)^{3 / 2}$.

It is straightforward to solve these equations. In Fig. 6 we show the yields of $V$ and $N$ as function of the inverse temperature for $m_{V}=10 \mathrm{MeV}, m_{N_{1}}=10 \mathrm{keV}$ and $g_{B-L}=$ $10^{-11.4}$.

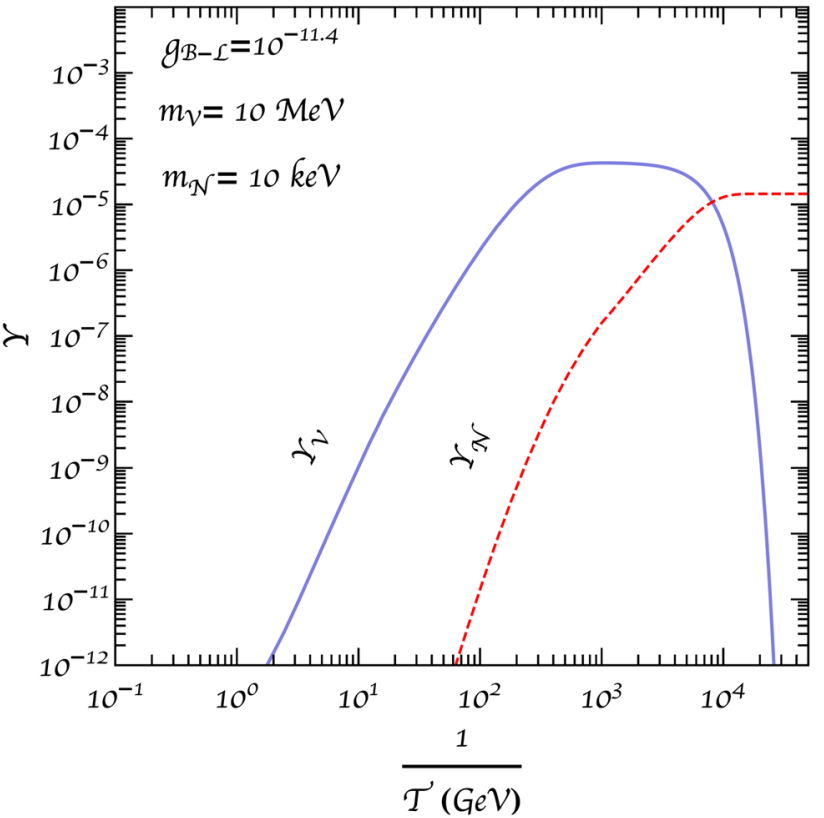

Fig. $6 V$ and $N_{1}$ yields as a function of the inverse temperature for $m_{V}=10 \mathrm{MeV}$ and $g_{B-L}=10^{-11.4}$

The resulting abundance of $N_{1}$ is

$\Omega_{N_{1}} h^{2} \equiv \frac{s_{0} m_{N_{1}}}{\rho_{c} h^{-2}} Y_{N_{1}} \simeq 2.7 \times 10^{2} Y_{N_{1}} \frac{m_{N_{1}}}{\mathrm{keV}}$,

where $s_{0}=2889.2 \mathrm{~cm}^{-3}$ is the entropy today and $\rho_{c}=$ $1.0510^{-5} h^{2} \mathrm{GeV} \mathrm{cm}^{-3}$ is the critical density. The evolution of $\Omega_{N_{1}} h^{2}$ is shown in Fig. 7 for two values of $m_{V}$ and a fixed value of $g_{B-L}$. Requiring that $\Omega_{N_{1}} h^{2}$ equals the full DM contribution of $\Omega_{D M} h^{2} \simeq 0.12$ implies a relation between $m_{V}$ and $g_{B-L}$ as shown in the curves of Fig. 2. The values of $g_{B-L}$ corresponding to the right dark matter relic abundance do not affect leptogenesis and lie far below the actual collider limits. Nevertheless some regions of the parameter space are interestingly excluded from supernova and BBN observations.

A final comment concerns the comparison of our calculation of the DM abundance and that in Ref. [67]. In this reference only the evolution of the $N_{1}$ is considered, and the collision term corresponds to the scattering process $f \bar{f} \rightarrow N_{1} N_{1}$, where the narrow width approximation is assumed. We believe this method is only equivalent to ours when all $f, \bar{f}$ and $V$ distributions are the equilibrium ones, but this is not the case here. In the region they can be compared our results are roughly a factor three smaller than those in [67].

\subsection{Couplings}

According to the previous calculation, the relic DM abundance requires a very small $B-L$ coupling. In order to 


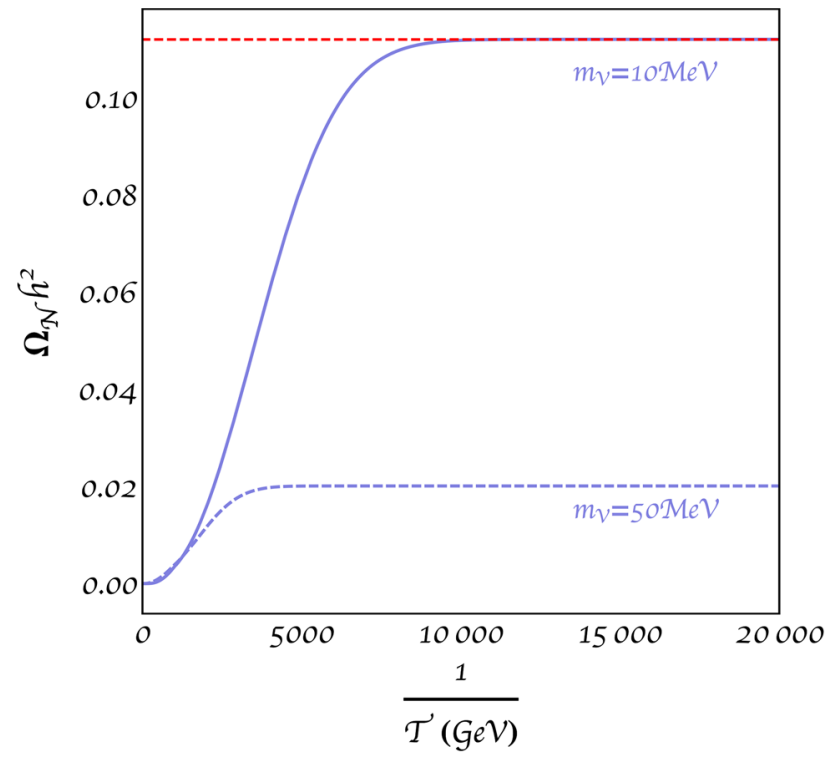

Fig. 7 The evolution of the density $\Omega_{N} h^{2}$ of the sterile neutrino $N_{1}$ in the $B-L$ model as a function of $1 / T(\mathrm{GeV})$. Here we have fixed $m_{N_{1}}=$ $10 \mathrm{keV}, g_{B-L}=10^{-11.4}$, and used two different value of the boson mass: $m_{V}=10,50 \mathrm{MeV}$. The gray line indicates the experimental value for dark matter abundance today

obtain, for example, a mass $m_{V} \sim 1 \mathrm{MeV}$, the gauge coupling needed to generate DM is

$g_{B-L} \sim 10^{-11.8}$,

and therefore

$\langle\phi\rangle \sim 2 \cdot 10^{8} \mathrm{GeV}$

In order to get the $N_{1}$ and $N_{2,3}$ in the target range of $\mathrm{keV}$ and $1-100 \mathrm{GeV}$ respectively, small and hierarchical $h_{N_{i}}$ couplings are needed:

$h_{N_{2}} \simeq h_{N_{3}} \sim 10^{-6}-10^{-8}$,

for the heavy sterile neutrinos involved in BO leptogenesis and

$h_{N_{1}} \sim 10^{-12}$,

for the dark matter candidate. Note that the required $h_{N}$ 's couplings are in the same ballpark as the yukawa couplings. The gauged $B-L$ model works nicely to explain neutrino masses, the baryon asymmetry and dark matter. Unfortunately it also requires a very small $g_{B-L}$ which will be very hard to test experimentally. An alternative might be to consider a flavoured $U(1)$, for example $L_{\mu}-L_{\tau}$, that might be compatible with a larger $g_{B-L}$, provided the assignment of charges to the singlet states ensures that not all of them reach thermalization via the flavoured gauge interaction before $t_{E W}$.

\section{Axion and neutrinos}

As a second example we consider an extension of Eq. (1) with a scalar doublet and a scalar singlet. This model is also an extension of the invisible axion model [87] with sterile neutrinos, that was first considered in [46], providing a connection between the Peccei-Quinn (PQ) symmetry breaking scale and the seesaw scale of the neutrino masses. The model contains two scalar doublets, $\Phi_{i}$, and one singlet, $\phi$. A $U(1)_{P Q}$ global symmetry exists if the two Higgs doublets couple separately to the up and down quarks and leptons so that the Yukawa Lagrangian takes the form:

$$
\begin{aligned}
& \mathcal{L} \supset-Y_{u} \bar{Q}_{L} \Phi_{1} u_{R}-Y_{d} \bar{Q}_{L} \Phi_{2} d_{R}-Y \bar{L}_{L} \Phi_{1} N \\
& \quad-Y_{l} \bar{L}_{L} \Phi_{2} l_{R}-\frac{h_{N}}{\sqrt{2}} \overline{N^{c}} N \phi+\text { h.c. }
\end{aligned}
$$

leading naturally to type II two-Higgs-doublet models without FCNC $[88,89]$.

The most general scalar potential of the model compatible with a global $U(1)_{P Q}$ is the following

$$
\begin{aligned}
V= & m_{1}^{2}\left|\Phi_{1}\right|^{2}+m_{2}^{2}\left|\Phi_{2}\right|^{2}+m^{2}|\phi|^{2} \\
& +\frac{\lambda_{1}}{2}\left|\Phi_{1}\right|^{4}+\frac{\lambda_{2}}{2}\left|\Phi_{2}\right|^{4}+\lambda_{3}\left|\Phi_{1}\right|^{2}\left|\Phi_{2}\right|^{2} \\
& +\lambda_{4}\left(\Phi_{1}^{\dagger} \Phi_{2}\right)\left(\Phi_{2}^{\dagger} \Phi_{1}\right) \\
& +\frac{\lambda_{\phi}}{2}|\phi|^{4}+\lambda_{1 \phi}\left|\Phi_{1}\right|^{2}|\phi|^{2}+\lambda_{2 \phi}\left|\Phi_{2}\right|^{2}|\phi|^{2} \\
& +k\left(\Phi_{1}^{\dagger} \Phi_{2}\right) \phi^{2} .
\end{aligned}
$$

The couplings in this potential can be chosen such that $\phi$ gets an expectation value,

$$
\langle\phi\rangle=\frac{1}{\sqrt{2}} f_{a},
$$

$U(1)_{P Q}$ is then spontaneously broken and a Nambu-Goldstone boson appears, the QCD axion. Furthermore the Majorana singlets $N$ get a mass. Expanding around the right vacuum, the field can be writen as

$\phi=\frac{1}{\sqrt{2}}\left(f_{a}+\sigma+i a\right)$,

where $\sigma$ is a massive field, while $a$ is the axion. Therefore after symmetry breaking we obtain an interaction term between sterile neutrinos and axions

$\mathcal{L} \supset-\frac{i h_{N}}{2} a \overline{N^{c}} N+$ h.c.

The breaking scale $f_{a}$ must be much larger than the vacuum expectation values of the doublets, $\gg v_{1,2}$, so that the axion can evade the stringent bounds from rare meson decays and supernova cooling, which sets a stringent lower bound $f_{a} \geq$ $4 \cdot 10^{8} \mathrm{GeV}[90]$. 
The mass of the axion is induced by the QCD anomaly in the sub-eV range:

$m_{a} \simeq \frac{z^{1 / 2}}{1+z} \frac{m_{\pi} f_{\pi}}{\langle\phi\rangle}$,

where $z=m_{u} / m_{d}$. For $f_{a} \geq 4 \cdot 10^{8} \mathrm{GeV}$, we have

$m_{a} \leq \mathcal{O}\left(10^{-2}\right) \mathrm{eV}$.

It is well known that the invisible axion is a viable cold DM candidate, through the misalignment mechanism $[87,91,92]$ (for recent reviews see [93,94]). The DM energy density is given by

$\Omega_{a} h^{2} \sim 2 \cdot 10^{4}\left(\frac{f_{a}}{10^{16} \mathrm{GeV}}\right)^{7 / 6}\left\langle\theta_{0}\right\rangle^{2}$,

where $\theta_{0}$ is the misalignment angle. The constraints on $f_{a}$ depend on whether the breaking of the PQ symmetry happens before or after inflation; in the latter case the misalignment angle can be averaged over many patches

$\left\langle\theta_{0}\right\rangle^{2} \sim \frac{\pi^{2}}{3}$,

so $\Omega_{a} \leq \Omega_{\mathrm{DM}}$ implies

$f_{a} \lesssim 1.2 \cdot 10^{11} \mathrm{GeV}$,

with the equality reproducing the observed cold dark matter energy density $\Omega_{\mathrm{CDM}} h^{2} \sim 0.12$. This correspond to the solid line in Fig. 8. If the PQ symmetry is broken before inflation, $\theta_{0}$ is a free parameter and the value of $f_{a}$ to account for DM is inversely proportional to $\theta_{0}^{2}$.

The axion can also manifest itself as dark radiation [95], given that it is also thermally produced [96]. This population of hot axions contributes to the effective number of relativistic species, but the size of this contribution is currently well within the observational bounds [97].

In this model the VEV of the scalar singlet gives a Majorana mass to the sterile neutrinos:

$m_{N} \approx h_{N} f_{a}$.

So, if we want a mass in the electroweak range, $\mathcal{O}\left(1-10^{2}\right)$ $\mathrm{GeV}$ and $f_{a} \in\left[10^{8}, 10^{11}\right] \mathrm{GeV}$, we need the coupling $h_{N}$ to be in the range:

$h_{N} \in\left[10^{-11}, 10^{-6}\right]$.

The hierarchy between $f_{a}$ and the electroweak scale requires that some couplings in the scalar potential in Eq. (44) $\left(k, \lambda_{1 \phi}, \lambda_{2 \phi}\right)$ are very small. Even if not very appealing theoretically, these small numbers are technically natural as already pointed out in [98], where the authors studied the same model with very heavy sterile neutrinos.

A relevant question is that of naturalness or fine-tunning of the Higgs mass in this model. In [98], this issue was studied in the context of high-scale thermal leptogenesis, and it was

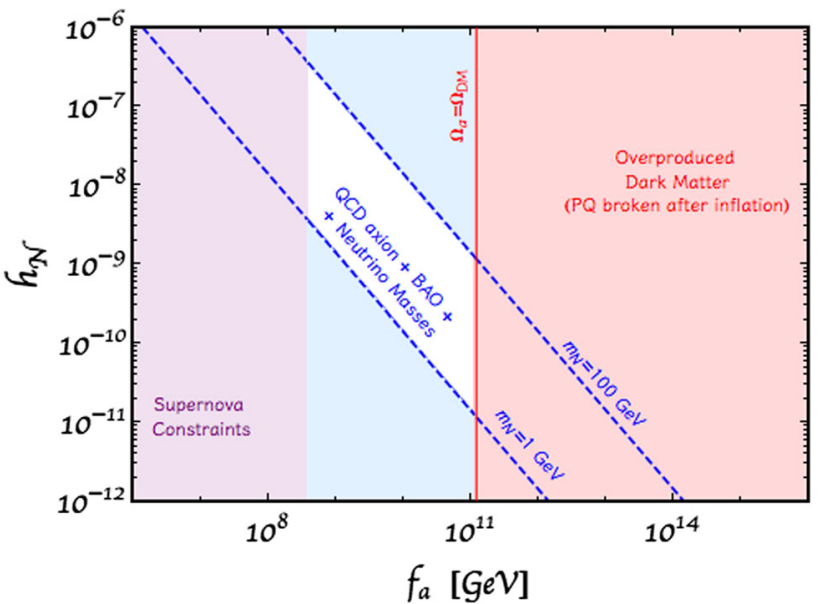

Fig. 8 The light purple region is forbidden by supernova cooling constraints (left band) and the red one by axions over closing the universe (right band). Axions can explain the DM relic density in the vertical line, if inflation happens before PQ symmetry breaking. Within the remaining parameter space, in the region between the two dashed lines successful leptogenesis via oscillations is possible

concluded that stability imposes relevant constraints. In particular, a relatively small $v_{2} \lesssim 30 \mathrm{GeV}$ is necessary to ensure viable leptogenesis for lower $m_{N}=10^{5}-10^{6} \mathrm{GeV}$ so that yukawa's are small enough, $y \leq 10^{-4}$, and do not induce unnaturally large corrections to the Higgs mass. In our case, the yukawa couplings, Eq. (5), are too small to give large corrections to the Higgs mass, so no additional constraint needs to be imposed on $v_{2}$. As a consequence other invisible axion models, such as the KSVZ [99,100], would also work in the context of low-scale $m_{N}$, but leads to tension with stability bounds in the high-scale version [101,102].

\subsection{Baryon asymmetry}

The possibility to generate the baryon asymmetry in this model a la Fukugita-Yanagida for very heavy neutrinos $m_{N} \sim f_{a}$ was recognized in the original proposal [46] and further elaborated in [98]. We want to point out here that for much smaller values of $h_{N}$, the BO mechanism could also work successfully.

As explained above the crucial point is whether the new interactions of the sterile states in this model are fast enough to equilibrate all the sterile neutrinos before $\mathrm{EW}$ phase transition. The leading order process we have to consider is the decay of the scalar into two sterile neutrinos $\sigma \rightarrow N N$, exactly as we considered in the previous section. The limit of $M_{\sigma} \sim f_{a} \geq 2 \times 10^{5}-5 \times 10^{6} \mathrm{GeV}$ derived in Eq. (15) also applies here, which is safely satisfied given the supernova cooling bounds.

At second order, we must also consider the new annihilation process of sterile neutrinos to axions $N N \leftrightarrow a a$ as shown in Fig. 9. The rate of this process at high tempera- 




Fig. 9 Annihilations of sterile neutrinos into Majorons

tures, $T \gg m_{N}$, is given by

$\Gamma_{N_{a}}=\frac{T^{3} m_{N}^{2}}{192 \pi f_{a}^{4}}$.

The condition $\Gamma_{N_{a}}(T)<H(T)$ is satisfied for $T \leq f_{a}$ if

$f_{a} \geq 1.2 \cdot 10^{5}\left(\frac{m_{N}}{1 \mathrm{GeV}}\right)^{2 / 3} \mathrm{GeV}$

(for $m_{N} \in\left[1,10^{2}\right] \mathrm{GeV}$ ), safely within the targeted range. Fig. 8 shows the region on the $\left(f_{a}, h_{N}\right)$ plane for which successful baryogenesis through the BO mechanism and DM can work in this model.

Even if the necessary condition for BO leptogenesis is met for $f_{a} \geq 10^{8} \mathrm{GeV}$, the presence of the extra degrees of freedom, the axion, the heavy scalar and the second doublet could modify quantitatively the baryon asymmetry. For example, the presence of two scalar doublets could modify the scattering rates of the sterile neutrinos considered in the BO scenario, where the main contributions [35,50,52,103] are:

$-2 \leftrightarrow 2$ scatterings on top quarks via higgs exchange

$-2 \leftrightarrow 2$ scatterings on gauge bosons

$-1 \leftrightarrow 2$ decays or inverse decays including resumed softgauge interactions

Sterile neutrinos are coupled to the same Higgs doublet that also couples to the top quarks; in this case nothing changes with respect to the usual calculation, in which the reactions with top quarks are mediated by $\Phi_{1}$. However, an alternative model, with a different $U(1)_{P Q}$ charge assignment, is also possible, in which the sterile neutrinos couple to $\phi_{2}$ and not $\phi_{1}$, as done in [98]. In this case top quark scattering does not contribute to sterile neutrino production at tree level, but the baryon asymmetry is not expected to change significantly, since the scattering rate on gauge bosons and the $1 \rightarrow 2$ processes are equally important $[50,52]$.

The process $\sigma \rightarrow N N$ is not foreseen to be relevant for $M_{\sigma} \sim f_{a}$, since the scalar is long decoupled when the generation of the asymmetry starts, while the new process $N N \leftrightarrow a a$ is expected to be very small according to the above estimates. It could nevertheless be interesting to look for possible corners of parameter space where the differences with respect to the minimal model is not negligible since this could provide a testing ground for the axion sector of the model.

\section{Majoron model}

In between the two models described in 3-4, there is the possibility of having a global $U$ (1) spontaneously broken, which is not related to the strong $\mathrm{CP}$ problem and we call it lepton number. This is of course the well-known singlet majoron model $[47,48,104]$. We assume the sterile neutrinos carry lepton number, $L_{N}=1$, but Majorana masses are forbidden and replaced by a yukawa interaction as in the $B-L$ model:

$\mathcal{L} \supset-\left(\bar{L} Y N \Phi+\frac{1}{\sqrt{2}} h_{N} \overline{N^{c}} N \phi+\right.$ h.c. $)$

where $\Phi$ is the standard model Higgs doublet, while $\phi$ is a complex scalar which carries lepton number $L_{\phi}=-2$. Then, the complex scalar acquires a VEV

$\phi=\frac{f+\sigma+i \eta}{\sqrt{2}}$

and the $U(1)_{L}$ is spontaneously broken giving rise to the right-handed Majorana mass matrix and leading to a Goldstone boson $\eta$, the majoron. Consequently the Lagrangian will induce the new scattering processes for neutrinos depicted in Fig. 9.

As usual we have to ensure that at least one sterile neutrino does not equilibrate before $T_{E W}$ (see $[105,106]$ for a recent discussion in the standard high-scale or resonant leptogenesis). As in the previous cases we have to consider the decay $\sigma \rightarrow N_{i} N_{i}$ and the annihilation into majorons, Fig. 9. The former gives the strongest constraint, as in Eq. (15):

$M_{\sigma} \sim f \geq 2 \times 10^{5}-5 \times 10^{6} \mathrm{GeV}$.

These lower bounds for $m_{N}=1$ and $100 \mathrm{GeV}$ are shown by the horizontal lines in Fig. 10.

\subsection{Dark matter}

There are two candidates in this model for dark matter that we consider in turn: the Majoron and the lightest sterile neutrino, $N_{1}$.

\subsubsection{Majoron}

In this model a natural candidate for dark matter is the majoron itself, but it has to acquire a mass, therefore becoming a pseudo Nambu-Goldstone boson (pNGB). One possibility is to appeal to gravitational effects $[107,108]$. However, the contribution to the mass from gravitational instantons is estimated to be [109-111]

$m_{\eta} \sim M_{P} e^{-\frac{M_{P}}{f}}$

and therefore extremely tiny, unless $f$ is close to the Planck scale. 




Fig. 10 Constraints in the plane $f-m_{\eta}$ considering the Majoron as the only dark matter component. The region in gray below the dashed line is excluded from CMB measurements [115], while the light red region below the solid (dashed) line is excluded in order not to spoil ARS for $M_{N}=100(1) \mathrm{GeV}$

Another alternative is to consider a flavoured $U(1)_{X}$ and soft symmetry breaking terms in the form of yukawa couplings $[112,113]$. This possibility has been studied in detail in Ref. [113]. It has been shown that the majoron can be the main component of dark matter for sterile neutrino masses $m_{N} \geq 10^{5} \mathrm{GeV}$, while for masses in the range we are interested in $\left(m_{N} \sim 1--100 \mathrm{GeV}\right)$ neither thermal production via freeze-out nor via freeze-in works.

The possibility to produce it via vacuum misalignment, analogous to the one which produces the axion relic density has also been discussed in [113]. It was shown to give a negligible contribution compared to the thermal one, because the majoron gets a temperature dependent mass at early times. Even if the mass of the majoron is significantly smaller in our situation, with lighter $m_{N}$, we find the same result, ie. that only a small fraction of the DM can be produced via misalignmet.

No matter what the production mechanism is if the majoron constitutes the dark matter, there are constraints from the requirement that the majoron be stable on a cosmological timescale and its decay to the light neutrinos

$\Gamma(\eta \rightarrow v v)=\frac{1}{64 \pi} \frac{\sum_{i} m_{v_{i}}^{2}}{f^{2}} m_{\eta}$

should not spoil the CMB anisotropy spectrum $[114,115]$. This gives constraints on the mass $m_{\eta}$ and the symmetry breaking scale $f$, as showed in Fig.10.

As in the axion case there are additional constraints from supernova cooling [116], but they are much weaker and give an upper bound much lower than the range shown in Fig. 10 . In the unconstrained region in Fig. 10, ARS leptogenesis and majoron DM could in principle work provided the mechanism to generate the majoron mass does not involve further interactions of the sterile neutrinos.

\subsubsection{Sterile neutrino}

We want now to consider the sterile neutrino as a dark matter candidate, a possibility already explored in $[117,118]$ in a model with a real scalar field and therefore no Majoron. In our case the presence of the Majoron could make the sterile neutrino unstable, given that it would decay through the channel

$\Gamma(N \rightarrow v \eta)=\frac{1}{32 \pi}\left(\frac{m_{N}}{f}\right)^{2} m_{v}$

Thus one has to assume that the Majoron has a larger mass so that this decay is kinematically forbidden.

As in the $B-L$ case, BO leptogenesis is driven by the other two heavier neutrino states $N_{2,3}$, while $N_{1}$ can be produced through freeze-in from $\sigma \rightarrow N_{1} N_{1}$ decay. Assuming $\sigma$ is in thermal equilibrium with the bath the Boltzmann equation describing the evolution of the $N_{1}$ density:

$\dot{n}_{1}+3 H n_{1}=2 \frac{M_{\sigma}^{2} \Gamma_{\sigma \rightarrow N_{1} N_{1}}}{2 \pi^{2}} T K_{1}\left(\frac{M_{\sigma}}{T}\right)$,

where we have neglected Pauli blocking, and the inverse processes.

Following the standard procedure we end with the contribution to the abundance:

$\Omega_{N_{1}} h^{2} \sim \frac{10^{27}}{g_{*}^{3 / 2}} \frac{m_{N_{1}} \Gamma_{\sigma \rightarrow N_{1} N_{1}}}{M_{\sigma}^{2}}$.

Using

$\Gamma_{\sigma \rightarrow N_{1} N_{1}}=\frac{h_{N}^{2} M_{\sigma}}{16 \pi}\left(1-\frac{4 m_{N_{1}}^{2}}{M_{\sigma}^{2}}\right) \sim \frac{h_{N}^{2} M_{\sigma}}{16 \pi}$,

and requiring that $\Omega_{N_{1}} h^{2}$ matches the observed DM we find

$h_{N} \sim 4.3 \cdot 10^{-13} \sqrt{\frac{m_{N_{1}}}{M_{\sigma}}}\left(\frac{g_{*}\left(m_{N_{1}}\right)}{10}\right)^{3 / 4}$.

The mass of the DM candidate is related to the coupling which regulates the freeze-in process through the VEV of $\phi$

$m_{N_{1}}=h_{N_{1}}\langle\phi\rangle$,

therefore if $M_{\sigma} \sim\langle\phi\rangle$, the coupling needs to be

$h_{N_{1}} \sim 10^{-9}-10^{-8}$,

as shown in Fig. 11. 


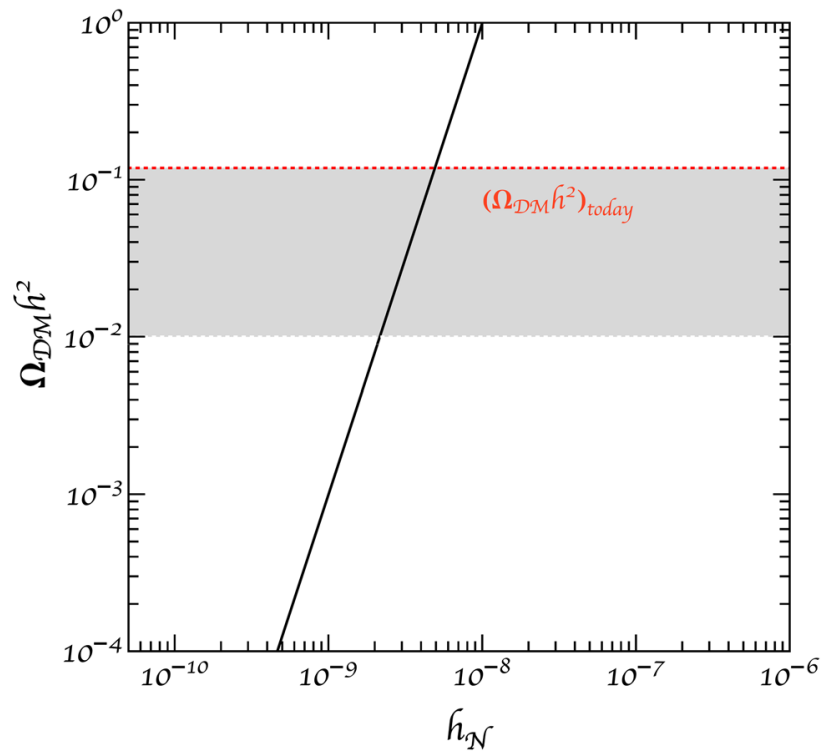

Fig. 11 Dark matter relic density as a function of the Yukawa coupling $h_{N}$ of the lightest sterile neutrino. The gray region identifies $0.01<\Omega_{D M} h^{2}<0.12$, that is to say a sterile neutrino contribution to DM density between 10 and $100 \%$. Taking $\langle\Phi\rangle \sim \mathrm{TeV}$, this region corresponds to $m_{N_{1}} \sim k e V$

If $m_{N_{1}}$ is the keV range so that it can satisfy cosmological and astrophysical constraints, the scale of the VEV should be

$\langle\phi\rangle \sim \mathrm{TeV}$.

As a consequence we see that if one couples the $B-L$ scalar also to the heavier neutrinos, the interactions are too fast and the BO mechanism cannot work since the bound in Eq. (59) is not satisfied. Alternatively, if we assume Eq. (59), then

$m_{N} \sim \mathcal{O}(\mathrm{MeV})$.

Such a massive neutrino would need extremely small activesterile mixing angle (collectively labeled $\theta$ ) to be sufficiently long-lived. The strongest bound from X-rays [119] give

$\sin ^{2}(2 \theta) \lesssim$ few $\times 10^{-6} \quad, m_{N} \sim \mathrm{keV}$,

while the soft gamma ray bound [120] gives

$\sin ^{2}(2 \theta) \lesssim$ few $\times 10^{-21}, \quad m_{N} \sim \mathrm{MeV}$.

In conclusion, either we consider a global symmetry with different family charges, more concretely a $(B-L)_{1}$, or we need to require extremely tiny yukawa coupling for the DM sterile neutrino, making this last model theoretically unappealing.

\section{Conclusion}

The extension of the Standard Model with three heavy majorana singlets at the weak scale can explain neutrino masses and also account for the baryon asymmetry in the Universe via the oscillation mechanism $[8,9]$. This scenario could be testable in future experiments. Unfortunately the simplest model cannot easily accommodate dark matter. In the $v$ MSM [9], one of the three heavy states is in the $\mathrm{keV}$ range and provides a candidate for dark matter, but it requires huge lepton asymmetries that cannot be naturally achieved in the minimal setup.

In this paper we have explored three extensions of the minimal scenario that can accommodate dark matter without spoiling baryogenesis. This is non trivial because new interactions of the heavy singlets can disrupt the necessary out-of-equilibrium condition which is mandatory to generate a lepton asymmetry. We have shown that a extension of the minimal model with a $U(1)_{B-L}$ gauge interaction can achieve this goal. The two heavier majorana fermions take part in the generation of the baryon asymmetry, while the lighest one in the $\mathrm{keV}$ range, $N_{1}$, is the dark matter. In contrast with the $v \mathrm{MSM}$ the production of the dark matter is not via mixing, but it is dominated by the $B-L$ gauge boson decay. The mixing is however what controls the decay of the $N_{1}$ and can be made sufficiently small to avoid the stringent $\mathrm{X}$-ray constraints. The correct DM abundance is achieved for very small $B-L$ gauge couplings, $g_{B-L} \lesssim 10^{-8}$, which are safely small not to disturb the baryon asymmetry, which remains the same as in the minimal model. Such tiny couplings are far below the reach of colliders. Supernova and $\mathrm{BBN}$ provide the most stringent constraints in the relevant region of parameter space, while future searches in SHIP might have a chance to touch on it.

We have also considered an extension involving an invisible axion sector with an extra scalar doublet and a complex singlet. The heavy majorana singlets get their mass from the PQ breaking scale [46]. DM is in the form of cold axions, from the misalignment mechanism and as is well known, the right relic abundance can be achieved for a large value of the PQ breaking scale, $f_{a} \simeq 10^{11} \mathrm{GeV}$. We have shown that such large scale is compatible with having the heavy neutrinos in the 1-100 GeV scale, and ARS leptogenesis. Finally we have considered the singlet majoron extension of the minimal model, with a global $U(1)_{B-L}$, that contains two potential DM candidates, the majoron or the lightest heavy neutrino, $N_{1}$. Unperturbed ARS baryogenesis requires a relatively high $B-L$ breaking scale, $f \gtrsim 10^{6} \mathrm{GeV}$. Majoron DM requires exotic production scenarios, while neutrino DM works for masses around $\mathrm{MeV}$, which requires extremely small mixings to make it sufficiently long-lived, or alternatively a less theoretically appealing possibity, where the scalar couples to 
only one sterile neutrino, while the other two have tree level masses or couple to a different scalar with a larger VEV.

As a general rule, adding new interactions that affect the heavy Majorana singlets modifies leptogenesis in the minimal model and viable extensions that can explain DM are likely to involve the freeze-in mechanism as in the examples above.

Acknowledgements We thank M. Escudero, M. Kekic, J. LópezPavón and J. Salvado for useful discussions. We acknowledge support from national grants FPA2014-57816-P, FPA2017-85985-P and the European projects H2020-MSCA-ITN-2015//674896-ELUSIVES and H2020-MSCA-RISE-2015

Data Availability Statement This manuscript has no associated data or the data will not be deposited. [Authors' comment: We didn't use real data in this paper, which is mostly a theoretical one. The only data we analyzed were taken from other works and are shown in the constraints plots.]

Open Access This article is distributed under the terms of the Creative Commons Attribution 4.0 International License (http://creativecomm ons.org/licenses/by/4.0/), which permits unrestricted use, distribution, and reproduction in any medium, provided you give appropriate credit to the original author(s) and the source, provide a link to the Creative Commons license, and indicate if changes were made.

Funded by SCOAP ${ }^{3}$.

\section{A Computation of momentum averaged rates in gauged $B-L$}

In this appendix we give some details on the computation of the momentum averaged rates in Eq. (24). The amplitude for $\bar{f} f \rightarrow N N$ for vanishing masses is given by

$$
\begin{aligned}
\sum_{\text {spins }}|\mathcal{M}|^{2} & =4 g_{B-L}^{4} \sum_{f} Q_{f}^{2} N_{c}\left[\frac{t^{2}+u^{2}}{s^{2}}\right] \\
& \equiv A\left[\frac{t^{2}+u^{2}}{s^{2}}\right],
\end{aligned}
$$

while that for $V V \rightarrow N N$, in the limit $M_{\sigma}^{2} \gg s, t \gg$ $m_{N}^{2}, m_{V}^{2}$ is

$$
\sum_{\text {spins }}|\mathcal{M}|^{2}=4 g_{B-L}^{4} \frac{m_{N}^{2} s}{m_{V}^{4}} .
$$

Defining the Bose-Einstein and Fermi-Dirac distributions

$$
f_{B}(x)=\frac{1}{e^{x}-1}, \quad f_{F}(x)=\frac{1}{e^{x}+1},
$$

and the variables

$q_{ \pm} \equiv \frac{1}{2}\left(q_{0} \pm|\mathbf{q}|\right)$

where $q=p_{1}+p_{2}$. We express all momenta in units of temperature $T$.
Following the procedure of Ref. [50] the rate $f \bar{f} \rightarrow N N$ can be writen as

$R^{(1)}(k)=\frac{A}{4(2 \pi)^{3} k_{0}}\left(r_{1}(k)+r_{2}(k)+r_{3}(k)\right)$,

with

$$
\begin{aligned}
r_{1}(k) \equiv & \int_{k_{0}}^{\infty} d q_{+} \int_{0}^{k_{0}} d q_{-} f_{B}\left(q_{+}+q_{-}\right) I_{1}\left(q_{+}, q_{-}\right), \\
r_{2}(k) \equiv & 2 \int_{k_{0}}^{\infty} d q_{+} \int_{0}^{k_{0}} d q_{-} f_{B}\left(q_{+}+q_{-}\right) \\
& \times \sum_{i=1,2} I_{i}\left(q_{+}, q_{-}\right) a_{i}\left[q_{+}, q_{-}, k_{0}\right] \\
r_{3}(k) \equiv & 2 \int_{k_{0}}^{\infty} d q_{+} \int_{0}^{k_{0}} d q_{-} f_{B}\left(q_{+}+q_{-}\right) \\
& \times \sum_{i=1,3} I_{i}\left(q_{+}, q_{-}\right) b_{i}\left[q_{+}, q_{-}, k_{0}\right]
\end{aligned}
$$

with

$I_{n}\left(q_{+}, q_{-}\right) \equiv \int_{q_{-}}^{q_{+}} x^{n-1}\left[1-2 f_{F}(x)\right] d x$,

and

$$
\begin{aligned}
a_{1}\left[q_{+}, q_{-}, k_{0}\right] \equiv & -1+\frac{q_{+}\left(k_{0}-q_{-}\right)+q_{-}\left(k_{0}-q_{+}\right)}{\left(q_{+}-q_{-}\right)^{2}} \\
a_{2}\left[q_{+}, q_{-}, k_{0}\right] \equiv & \frac{q_{+}+q_{-}-2 k_{0}}{\left(q_{+}-q_{-}\right)^{2}}, \\
b_{1}\left[q_{+}, q_{-}, k_{0}\right] \equiv & a_{1}^{2}+2 q_{+} q_{-} \frac{\left(q_{+}-k_{0}\right)\left(q_{-}-k_{0}\right)}{\left(q_{+}-q_{-}\right)^{4}} b_{2}\left[q_{+}, q_{-}, k_{0}\right] \\
\equiv & 2 a_{1}\left[q_{+}, q_{-}, k_{0}\right] a_{2}\left[q_{+}, q_{-}, k_{0}\right]+ \\
& -2\left(q_{+}+q_{-}\right) \frac{\left(q_{+}-k_{0}\right)\left(q_{-}-k_{0}\right)}{\left(q_{+}-q_{-}\right)^{4}} \\
b_{3}\left[q_{+}, q_{-}, k_{0}\right] \equiv & a_{2}^{2}+2 \frac{\left(q_{+}-k_{0}\right)\left(q_{-}-k_{0}\right)}{\left(q_{+}-q_{-}\right)^{4}}
\end{aligned}
$$

The rate $V V \rightarrow N N$ can be written as

$$
\begin{aligned}
R^{(2)}(k)= & \frac{A^{\prime}}{(2 \pi)^{3} k_{0}} \int_{k_{0}}^{\infty} d q_{+} \int_{0}^{k_{0}} d q_{-}\left(q_{+} q_{-}\right) f_{B}\left(q_{+}+q_{-}\right) \\
& \times I_{1}^{\prime}\left(q_{+}, q_{-}\right),
\end{aligned}
$$

with

$I_{1}^{\prime}\left(q_{+}, q_{-}\right) \equiv \int_{q_{-}}^{q_{+}}\left[1+2 f_{B}(x)\right] d x$.

and $A^{\prime} \equiv g_{B-L}^{4} \frac{m_{N}^{2}}{m_{V}^{4}}$.

The total rate is

$$
R(k)=\sum_{i=1,2} R^{(i)}(k),
$$

and the averaged rates $\gamma_{V}^{(0)}$ and $\gamma_{V}^{(1)}$ are found to be:

$$
\left\langle\gamma_{V}^{(0)}\right\rangle=g_{B-L}^{4} T
$$




$$
\begin{aligned}
& \times\left(3.2(3) \times 10^{-3}+2.95 \times 10^{-4} \frac{m_{N}^{2} T^{2}}{m_{V}^{4}}\right) \\
\left\langle\gamma_{V}^{(1)}\right\rangle= & g_{B-L}^{4} T \\
& \times\left(3.4(1) \times 10^{-4}+3.55 \times 10^{-5} \frac{m_{N}^{2} T^{2}}{m_{V}^{4}}\right) .
\end{aligned}
$$

where

$$
\left\langle\gamma_{V}^{(0)}\right\rangle \equiv \frac{\int \frac{d^{3} k}{(2 \pi)^{3} 2 k_{0}} R[k]}{\int \frac{d^{3} k}{(2 \pi)^{3}} f_{F}(k)}
$$

and

$\left\langle\gamma_{V}^{(1)}\right\rangle \equiv \frac{\int \frac{d^{3} k}{(2 \pi)^{3} 2 k_{0}} R[k] f_{F}(k)}{\int \frac{d^{3} k}{(2 \pi)^{3}} f_{F}(k)}$

\section{References}

1. P. Minkowski, $\mu \rightarrow e \gamma$ at a Rate of One Out of $10^{9}$ Muon Decays? Phys. Lett. 67B, 421-428 (1977). https://doi.org/10. 1016/0370-2693(77)90435-X

2. M. Gell-Mann, P. Ramond, R. Slansky, Complex spinors and unified theories. Conf. Proc. C 790927, 315-321 (1979). arXiv: 1306.4669

3. T. Yanagida, Horizontal symmetry and masses of neutrinos. Conf. Proc. C 7902131, 95-99 (1979)

4. R.N. Mohapatra, G. Senjanovic, Neutrino Mass and Spontaneous Parity Violation. Phys. Rev. Lett. 44, 912 (1980). https://doi.org/ 10.1103/PhysRevLett.44.912

5. M. Fukugita, T. Yanagida, Baryogenesis without grand unification. Phys. Lett. B 174, 45-47 (1986). https://doi.org/10.1016/ 0370-2693(86)91126-3

6. S. Davidson, A. Ibarra, A Lower bound on the righthanded neutrino mass from leptogenesis. Phys. Lett. B 535, 25-32 (2002). https://doi.org/10.1016/S0370-2693(02)01735-5. arXiv:hep-ph/0202239

7. A. Abada, S. Davidson, A. Ibarra, F.X. Josse-Michaux, M. Losada, A. Riotto, Flavour matters in leptogenesis. JHEP 09, 010 (2006). https://doi.org/10.1088/1126-6708/2006/09/010. arXiv:hep-ph/0605281

8. E.K. Akhmedov, V.A. Rubakov, AYu. Smirnov, Baryogenesis via neutrino oscillations. Phys. Rev. Lett. 81, 13591362 (1998). https://doi.org/10.1103/PhysRevLett.81.1359. arXiv:hep-ph/9803255

9. T. Asaka, M. Shaposhnikov, The nuMSM, dark matter and baryon asymmetry of the universe. Phys. Lett. B 620, 17-26 (2005). https://doi.org/10.1016/j.physletb.2005.06.020. arXiv:hep-ph/0505013

10. V.A. Kuzmin, V.A. Rubakov, M.E. Shaposhnikov, On the anomalous electroweak baryon number nonconservation in the early universe. Phys. Lett. 155B, 36 (1985). https://doi.org/10.1016/ 0370-2693(85)91028-7

11. A. Ferrari, J. Collot, M.-L. Andrieux, B. Belhorma, P. de Saintignon, J.-Y. Hostachy, P. Martin, M. Wielers, Sensitivity study for new gauge bosons and right-handed Majorana neutrinos in $p p$ collisions at $s=14-\mathrm{TeV}$. Phys. Rev. D 62, 013001 (2000). https://doi.org/10.1103/PhysRevD.62.013001

12. M. L. Graesser, Experimental constraints on higgs boson decays to TeV-scale right-handed neutrinos. arXiv:0705.2190
13. F. del Aguila, J.A. Aguilar-Saavedra, Distinguishing seesaw models at LHC with multi-lepton signals. Nucl. Phys. B 813, 22-90 (2009). https://doi.org/10.1016/j.nuclphysb.2008.12.029. arXiv:0808.2468

14. P.S. Bhupal Dev, R. Franceschini, R.N. Mohapatra, Bounds on $\mathrm{TeV}$ seesaw models from LHC higgs data. Phys. Rev. D 86, 093010 (2012). https://doi.org/10.1103/PhysRevD.86.093010. arXiv: 1207.2756

15. J.C. Helo, M. Hirsch, S. Kovalenko, Heavy neutrino searches at the LHC with displaced vertices. Phys. Rev. D 89, 073005 (2014) [Erratum: Phys. Rev.D93,no.9,099902(2016)]. arXiv:1312.2900, https://doi.org/10.1103/PhysRevD.89.073005. https://doi.org/10.1103/PhysRevD.93.099902

16. A. Blondel, E. Graverini, N. Serra, M. Shaposhnikov, Search for heavy right handed neutrinos at the FCC-ee. Nucl. Part. Phys. Proc. 273-275, 1883-1890 (2016). https://doi.org/10.1016/ j.nuclphysbps.2015.09.304. arXiv:1411.5230

17. A. Abada, V. De Romeri, S. Monteil, J. Orloff, A.M. Teixeira, Indirect searches for sterile neutrinos at a high-luminosity Z-factory. JHEP 04, 051 (2015). https://doi.org/10.1007/JHEP04(2015)051. arXiv: 1412.6322

18. Y. Cui, B. Shuve, Probing baryogenesis with displaced vertices at the LHC. JHEP 02, 049 (2015). https://doi.org/10.1007/ JHEP02(2015)049. arXiv:1409.6729

19. S. Antusch, O. Fischer, Testing sterile neutrino extensions of the Standard Model at future lepton colliders. JHEP 05, 053 (2015). https://doi.org/10.1007/JHEP05(2015)053. arXiv:1502.05915

20. A.M. Gago, P. Hernandez, J. Jones-Perez, M. Losada, A. Moreno Briceño, Probing the Type I seesaw mechanism with displaced vertices at the LHC. Eur. Phys. J. C 75(10), 470 (2015). https:// doi.org/10.1140/epjc/s10052-015-3693-1. arXiv:1505.05880

21. S. Antusch, E. Cazzato, O. Fischer, Displaced vertex searches for sterile neutrinos at future lepton colliders. JHEP 12, 007 (2016). https://doi.org/10.1007/JHEP12(2016)007. arXiv:1604.02420

22. A. Caputo, P. Hernandez, M. Kekic, J. Lopez-Pavon, J. Salvado, The seesaw path to leptonic CP violation. Eur. Phys. J. C 77(4), 258 (2017). https://doi.org/10.1140/epjc/s10052-017-4823-8. arXiv: 1611.05000

23. A. Caputo, P. Hernandez, J. Lopez-Pavon, J. Salvado, The seesaw portal in testable models of neutrino masses. JHEP 06, 112 (2017). https://doi.org/10.1007/JHEP06(2017)112. arXiv:1704.08721

24. M. Shaposhnikov, The nuMSM, leptonic asymmetries, and properties of singlet fermions. JHEP 08, 008 (2008). https://doi.org/ 10.1088/1126-6708/2008/08/008. arXiv:0804.4542

25. L. Canetti, M. Drewes, M. Shaposhnikov, Matter and antimatter in the universe. New J. Phys. 14, 095012 (2012). https://doi.org/ 10.1088/1367-2630/14/9/095012. arXiv: 1204.4186

26. L. Canetti, M. Drewes, T. Frossard, M. Shaposhnikov, Dark matter, baryogenesis and neutrino oscillations from right handed neutrinos. Phys. Rev. D 87, 093006 (2013). https://doi.org/10.1103/ PhysRevD.87.093006. arXiv:1208.4607

27. T. Asaka, S. Eijima, H. Ishida, Kinetic equations for baryogenesis via sterile neutrino oscillation. JCAP 1202, 021 (2012). https:// doi.org/10.1088/1475-7516/2012/02/021. arXiv:1112.5565

28. B. Shuve, I. Yavin, Baryogenesis through neutrino oscillations: a unified perspective. Phys. Rev. D 89(7), 075014 (2014). https:// doi.org/10.1103/PhysRevD.89.075014. arXiv:1401.2459

29. A. Abada, G. Arcadi, V. Domcke, M. Lucente, Lepton number violation as a key to low-scale leptogenesis. JCAP 1511(11), 041 (2015). https://doi.org/10.1088/1475-7516/2015/ 11/041. arXiv: 1507.06215

30. P. Hernandez, M. Kekic, J. Lopez-Pavon, J. Racker, N. Rius, Leptogenesis in GeV scale seesaw models. JHEP 10, 067 (2015). https://doi.org/10.1007/JHEP10(2015)067. arXiv:1508.03676 
31. P. Hernandez, M. Kekic, J. Lopez-Pavon, J. Racker, J. Salvado, Testable Baryogenesis in Seesaw Models. JHEP 08, 157 (2016). https://doi.org/10.1007/JHEP08(2016)157. arXiv:1606.06719

32. M. Drewes, B. Garbrecht, D. Gueter, J. Klaric, Leptogenesis from oscillations of heavy neutrinos with large mixing angles. JHEP 12, 150 (2016). https://doi.org/10.1007/JHEP12(2016)150. arXiv: 1606.06690

33. M. Drewes, B. Garbrecht, D. Gueter, J. Klaric, Testing the low scale seesaw and leptogenesis. JHEP 08, 018 (2017). https://doi. org/10.1007/JHEP08(2017)018. arXiv:1609.09069

34. T. Hambye, D. Teresi, Higgs doublet decay as the origin of the baryon asymmetry. Phys. Rev. Lett. 117(9), 091801 (2016). https://doi.org/10.1103/PhysRevLett.117.091801. arXiv: 1606.00017

35. J. Ghiglieri, M. Laine, GeV-scale hot sterile neutrino oscillations: a derivation of evolution equations. JHEP 05, 132 (2017). https:// doi.org/10.1007/JHEP05(2017)132. arXiv:1703.06087

36. T. Asaka, S. Eijima, H. Ishida, K. Minogawa, T. Yoshii, Initial condition for baryogenesis via neutrino oscillation. arXiv:1704.02692

37. T. Hambye, D. Teresi, Baryogenesis from L-violating Higgsdoublet decay in the density-matrix formalism. Phys. Rev. D 96(1), 015031 (2017). https://doi.org/10.1103/PhysRevD.96. 015031. arXiv: 1705.00016

38. A. Abada, G. Arcadi, V. Domcke, M. Lucente, Neutrino masses, leptogenesis and dark matter from small lepton number violation? JCAP 1712(12), 024 (2017). https://doi.org/10.1088/1475-7516/ 2017/12/024. arXiv:1709.00415

39. J. Ghiglieri, M. Laine, GeV-scale hot sterile neutrino oscillations: a numerical solution. JHEP 02, 078 (2018). https://doi.org/10. 1007/JHEP02(2018)078. arXiv:1711.08469

40. S. Dodelson, L.M. Widrow, Sterile-neutrinos as dark matter. Phys. Rev. Lett. 72, 17-20 (1994). https://doi.org/10.1103/ PhysRevLett.72.17. arXiv:hep-ph/9303287

41. X.-D. Shi, G.M. Fuller, A New dark matter candidate: nonthermal sterile neutrinos. Phys. Rev. Lett. 82, 2832-2835 (1999). https:// doi.org/10.1103/PhysRevLett.82.2832. arXiv:astro-ph/9810076

42. K. Perez, K.C.Y. Ng, J.F. Beacom, C. Hersh, S. Horiuchi, R. Krivonos, Almost closing the $\mathrm{v}$ MSM sterile neutrino dark matter window with NuSTAR. Phys. Rev. D 95(12), 123002 (2017). https://doi.org/10.1103/PhysRevD.95.123002. arXiv: 1609.00667

43. J. Baur, N. Palanque-Delabrouille, C. Yeche, A. Boyarsky, O. Ruchayskiy, É. Armengaud, J. Lesgourgues, Constraints from Ly- $\alpha$ forests on non-thermal dark matter including resonantlyproduced sterile neutrinos. JCAP 1712(12), 013 (2017). https:// doi.org/10.1088/1475-7516/2017/12/013. arXiv:1706.03118

44. S. Baumholzer, V. Brdar, P. Schwaller, The New $v$ MSM: radiative neutrino masses, $\mathrm{keV}$-scale dark matter and viable leptogenesis with sub-TeV new physics. arXiv:1806.06864

45. R.N. Mohapatra, R.E. Marshak, Local B-L symmetry of electroweak interactions, majorana neutrinos and neutron oscillations. Phys. Rev. Lett. 44, 1316-1319 (1980) [Erratum: Phys. Rev. Lett.44,1643(1980)]. https://doi.org/10.1103/PhysRevLett. 44.1644.2. https://doi.org/10.1103/PhysRevLett.44.1316

46. P. Langacker, R.D. Peccei, T. Yanagida, Invisible axions and light neutrinos: are they connected? Mod. Phys. Lett. A 1, 541 (1986). https://doi.org/10.1142/S0217732386000683

47. Y. Chikashige, R.N. Mohapatra, R.D. Peccei, Are there real goldstone bosons associated with broken lepton number? Phys. Lett. 98B, 265-268 (1981). https://doi.org/10.1016/ 0370-2693(81)90011-3

48. J. Schechter, J.W.F. Valle, Neutrino decay and spontaneous violation of lepton number. Phys. Rev. D 25, 774 (1982). https://doi. org/10.1103/PhysRevD.25.774

49. M. Drewes, B. Garbrecht, P. Hernandez, M. Kekic, J. LopezPavon, J. Racker, N. Rius, J. Salvado, D. Teresi, A.R.S. Lepto- genesis, Int. J. Mod. Phys. A 33(05n06), 1842002 (2018). https:// doi.org/10.1142/S0217751X18420022. arXiv:1711.02862

50. D. Besak, D. Bodeker, Thermal production of ultrarelativistic right-handed neutrinos: complete leading-order results. JCAP 1203, 029 (2012). https://doi.org/10.1088/1475-7516/2012/03/ 029. arXiv: 1202.1288

51. B. Garbrecht, F. Glowna, P. Schwaller, Scattering Rates for leptogenesis: damping of lepton flavour coherence and production of singlet neutrinos. Nucl. Phys. B 877, 1-35 (2013). https://doi. org/10.1016/j.nuclphysb.2013.08.020. arXiv:1303.5498

52. I. Ghisoiu, M. Laine, Right-handed neutrino production rate at $T>160 \mathrm{GeV}$. JCAP 1412(12), 032 (2014). https://doi.org/10. 1088/1475-7516/2014/12/032. arXiv: 1411.1765

53. G. Bellini et al., Precision measurement of the $7 \mathrm{Be}$ solar neutrino interaction rate in Borexino. Phys. Rev. Lett. 107, 141302 (2011). https://doi.org/10.1103/PhysRevLett.107. 141302. arXiv: 1104.1816

54. S. Chatrchyan et al., Measurement of the differential and doubledifferential Drell-Yan cross sections in proton-proton collisions at $\sqrt{s}=7$ TeV. JHEP 12, 030 (2013). https://doi.org/10.1007/ JHEP12(2013)030. arXiv:1310.7291

55. J.P. Lees et al., Search for a dark photon in $e^{+} e^{-}$collisions at BaBar. Phys. Rev. Lett. 113(20), 201801 (2014). https://doi.org/ 10.1103/PhysRevLett.113.201801. arXiv: 1406.2980

56. J.P. Lees et al., Search for Invisible decays of a dark photon produced in $e^{+} e^{-}$collisions at BaBar. Phys. Rev. Lett. 119(13), 131804 (2017). https://doi.org/10.1103/PhysRevLett. 119.131804. arXiv: 1702.03327

57. V. Khachatryan et al., Search for physics beyond the standard model in dilepton mass spectra in proton-proton collisions at $\sqrt{s}=8$ TeV. JHEP 04, 025 (2015). https://doi.org/10.1007/ JHEP04(2015)025. arXiv:1412.6302

58. G. Aad et al., Search for high-mass dilepton resonances in pp collisions at $\sqrt{s}=8 \mathrm{TeV}$ with the ATLAS detector. Phys. Rev. D 90(5), 052005 (2014). https://doi.org/10.1103/PhysRevD.90. 052005. arXiv: 1405.4123

59. A Combination of preliminary electroweak measurements and constraints on the standard model. arXiv:hep-ex/0412015

60. T. Appelquist, B.A. Dobrescu, A.R. Hopper, Nonexotic neutral gauge bosons. Phys. Rev. D 68, 035012 (2003). https://doi.org/ 10.1103/PhysRevD.68.035012. arXiv:hep-ph/0212073

61. B. Batell, M. Pospelov, B. Shuve, Shedding light on neutrino masses with dark forces. JHEP 08, 052 (2016). https://doi.org/ 10.1007/JHEP08(2016)052. arXiv:1604.06099

62. M. Klasen, F. Lyonnet, F.S. Queiroz, NLO+NLL collider bounds, Dirac fermion and scalar dark matter in the B-L model. Eur. Phys. J. C 77(5), 348 (2017). https://doi.org/10.1140/epjc/ s10052-017-4904-8. arXiv:1607.06468

63. P. Ilten, Y. Soreq, M. Williams, W. Xue, Serendipity in dark photon searches. JHEP 06, 004 (2018). https://doi.org/10.1007/ JHEP06(2018)004. arXiv:1801.04847

64. M. Escudero, S. J. Witte, N. Rius, The dispirited case of gauged $U(1)_{B-L}$ dark matter. arXiv: 1806.02823

65. S. Alekhin et al., A facility to search for hidden particles at the CERN SPS: the SHiP physics case. Rept. Prog. Phys. 79(12), 124201 (2016). https://doi.org/10.1088/0034-4885/79/ 12/124201. arXiv: 1504.04855

66. D. Gorbunov, A. Makarov, I. Timiryasov, Decaying light particles in the SHiP experiment: signal rate estimates for hidden photons. Phys. Rev. D 91(3), 035027 (2015). https://doi.org/10.1103/ PhysRevD.91.035027. arXiv:1411.4007

67. K. Kaneta, Z. Kang, H.-S. Lee, Right-handed neutrino dark matter under the $B-L$ gauge interaction. JHEP 02, 031 (2017). https:// doi.org/10.1007/JHEP02(2017)031. arXiv:1606.09317 
68. J.H. Chang, R. Essig, S.D. McDermott, Revisiting supernova 1987A constraints on dark photons. JHEP 01, 107 (2017). https:// doi.org/10.1007/JHEP01(2017)107. arXiv:1611.03864

69. B. Ahlgren, T. Ohlsson, S. Zhou, Comment on "Is dark matter with long-range interactions a solution to all small-scale problems of cold dark matter cosmology?". Phys. Rev. Lett. 111(19), 199001 (2013). https://doi.org/10.1103/PhysRevLett. 111.199001. arXiv:1309.0991

70. M. Williams, C.P. Burgess, A. Maharana, F. Quevedo, New constraints (and Motivations) for Abelian Gauge Bosons in the MeVTeV mass range. JHEP 08, 106 (2011). https://doi.org/10.1007/ JHEP08(2011)106. arXiv:1103.4556

71. R. Mayle, J.R. Wilson, J.R. Ellis, K.A. Olive, D.N. Schramm, G. Steigman, Constraints on Axions from SN 1987a. Phys. Lett. B 203, 188-196 (1988). https://doi.org/10.1016/ 0370-2693(88)91595-X

72. G. Raffelt, D. Seckel, Bounds on exotic particle interactions from SN 1987a. Phys. Rev. Lett. 60, 1793 (1988). https://doi.org/10. 1103/PhysRevLett.60.1793

73. M.S. Turner, Axions from SN 1987a. Phys. Rev. Lett. 60, 1797 (1988). https://doi.org/10.1103/PhysRevLett.60.1797

74. J.D. Bjorken, R. Essig, P. Schuster, N. Toro, New fixedtarget experiments to search for dark Gauge forces. Phys. Rev. D 80, 075018 (2009). https://doi.org/10.1103/PhysRevD.80. 075018. arXiv:0906.0580

75. P. Vilain et al., Measurement of differential cross-sections for muon-neutrino electron scattering. Phys. Lett. B 302, 351-355 (1993). https://doi.org/10.1016/0370-2693(93)90408-A

76. J.D. Bjorken, S. Ecklund, W.R. Nelson, A. Abashian, C. Church, B. Lu, L.W. Mo, T.A. Nunamaker, P. Rassmann, Search for neutral metastable penetrating particles produced in the SLAC beam dump. Phys. Rev. D 38, 3375 (1988). https://doi.org/10.1103/ PhysRevD.38.3375

77. E.M. Riordan et al., A search for short lived axions in an electron beam dump experiment. Phys. Rev. Lett. 59, 755 (1987). https:// doi.org/10.1103/PhysRevLett.59.755

78. A. Bross, M. Crisler, S.H. Pordes, J. Volk, S. Errede, J. Wrbanek, A search for shortlived particles produced in an electron beam dump. Phys. Rev. Lett. 67, 2942-2945 (1991). https://doi.org/10. 1103/PhysRevLett.67.2942

79. A. Fradette, M. Pospelov, J. Pradler, A. Ritz, Cosmological constraints on very dark photons. Phys. Rev. D 90(3), 035022 (2014). https://doi.org/10.1103/PhysRevD.90.035022. arXiv:1407.0993

80. J. Berger, K. Jedamzik, D.G.E. Walker, Cosmological constraints on decoupled dark photons and dark higgs. JCAP 1611, 032 (2016). https://doi.org/10.1088/1475-7516/2016/11/ 032. arXiv: 1605.07195

81. G.-Y. Huang, T. Ohlsson, S. Zhou, Observational constraints on secret neutrino interactions from big bang nucleosynthesis. Phys. Rev. D 97(7), 075009 (2018). https://doi.org/10.1103/PhysRevD. 97.075009. arXiv: 1712.04792

82. J. Heeck, D. Teresi, Leptogenesis and neutral gauge bosons. Phys. Rev. D 94(9), 095024 (2016). https://doi.org/10.1103/PhysRevD. 94.095024. arXiv:1609.03594

83. H.A. Weldon, Effective Fermion masses of order gT in high temperature gauge theories with exact chiral invariance. Phys. Rev. D 26, 2789 (1982). https://doi.org/10.1103/PhysRevD.26.2789

84. G. Sigl, G. Raffelt, General kinetic description of relativistic mixed neutrinos. Nucl. Phys. B 406, 423-451 (1993). https://doi. org/10.1016/0550-3213(93)90175-O

85. J. McDonald, Thermally generated gauge singlet scalars as selfinteracting dark matter. Phys. Rev. Lett. 88, 091304 (2002). https://doi.org/10.1103/PhysRevLett.88.091304. arXiv:hep-ph/0106249
86. L.J. Hall, K. Jedamzik, J. March-Russell, S.M. West, Freeze-in production of FIMP dark matter. JHEP 03, 080 (2010). https:// doi.org/10.1007/JHEP03(2010)080. arXiv:0911.1120

87. M. Dine, W. Fischler, The Not So Harmless Axion. Phys. Lett. B 120, 137-141 (1983). https://doi.org/10.1016/ 0370-2693(83)90639-1

88. G.C. Branco, P.M. Ferreira, L. Lavoura, M.N. Rebelo, M. Sher, J.P. Silva, Theory and phenomenology of two-Higgs-doublet models. Phys. Rept. 516, 1-102 (2012). https://doi.org/10.1016/j.physrep. 2012.02.002. arXiv:1106.0034

89. D. Espriu, F. Mescia, A. Renau, Axion-Higgs interplay in the two Higgs-doublet model. Phys. Rev. D 92(9), 095013 (2015). https:// doi.org/10.1103/PhysRevD.92.095013. arXiv:1503.02953

90. G.G. Raffelt, Particle physics from stars. Ann. Rev. Nucl. Part. Sci. 49, 163-216 (1999). https://doi.org/10.1146/annurev.nucl. 49.1.163. arXiv:hep-ph/9903472

91. J. Preskill, M.B. Wise, F. Wilczek, Cosmology of the invisible axion. Phys. Lett. B 120, 127-132 (1983). https://doi.org/10. 1016/0370-2693(83)90637-8

92. L.F. Abbott, P. Sikivie, A cosmological bound on the invisible axion. Phys. Lett. B 120, 133-136 (1983). https://doi.org/10. 1016/0370-2693(83)90638-X

93. M. Kawasaki, K. Nakayama, Axions: theory and cosmological role. Ann. Rev. Nucl. Part. Sci. 63, 69-95 (2013). https://doi.org/ 10.1146/annurev-nucl-102212-170536. arXiv:1301.1123

94. D.J.E. Marsh, Axion cosmology. Phys. Rept. 643, 179 (2016). https://doi.org/10.1016/j.physrep.2016.06.005. arXiv: 1510.07633

95. S. Weinberg, Goldstone Bosons as fractional cosmic neutrinos. Phys. Rev. Lett. 110(24), 241301 (2013). https://doi.org/10.1103/ PhysRevLett.110.241301. arXiv:1305.1971

96. P. Graf, F.D. Steffen, Thermal axion production in the primordial quark-gluon plasma. Phys. Rev. D 83, 075011 (2011). https://doi. org/10.1103/PhysRevD.83.075011. arXiv:1008.4528

97. A. Salvio, A. Strumia, W. Xue, Thermal axion production. JCAP 1401, 011 (2014). https://doi.org/10.1088/1475-7516/2014/01/ 011. arXiv: 1310.6982

98. J.D. Clarke, R.R. Volkas, Technically natural nonsupersymmetric model of neutrino masses, baryogenesis, the strong CP problem, and dark matter. Phys. Rev. D 93(3), 035001 (2016). https://doi.org/10.1103/PhysRevD.93.035001. [Phys. Rev.D93,035001(2016)]. arXiv:1509.07243

99. J.E. Kim, Weak interaction singlet and strong CP invariance. Phys. Rev. Lett. 43, 103 (1979). https://doi.org/10.1103/PhysRevLett. 43.103

100. M.A. Shifman, A.I. Vainshtein, V.I. Zakharov, Can confinement ensure natural $\mathrm{CP}$ invariance of strong interactions? Nucl. Phys. B 166, 493-506 (1980). https://doi.org/10.1016/ 0550-3213(80)90209-6

101. G. Ballesteros, J. Redondo, A. Ringwald, C. Tamarit, Unifying inflation with the axion, dark matter, baryogenesis and the seesaw mechanism. Phys. Rev. Lett. 118(7), 071802 (2017). https://doi. org/10.1103/PhysRevLett.118.071802. arXiv:1608.05414

102. G. Ballesteros, J. Redondo, A. Ringwald, C. Tamarit, Standard Model axion seesaw Higgs portal inflation. Five problems of particle physics and cosmology solved in one stroke. JCAP 1708(08), 001 (2017). https://doi.org/10.1088/1475-7516/2017/ 08/001. arXiv:1610.01639

103. T. Asaka, M. Laine, M. Shaposhnikov, On the hadronic contribution to sterile neutrino production. JHEP 06, 053 (2006). https://doi.org/10.1088/1126-6708/2006/06/053. arXiv:hep-ph/0605209

104. J.M. Cline, K. Kainulainen, K.A. Olive, Constraints on majoron models, neutrino masses and baryogenesis. Astropart. Phys. 1, 387-398 (1993). https://doi.org/10.1016/ 0927-6505(93)90005-X. arXiv:hep-ph/9304229 
105. P.-H. Gu, U. Sarkar, Leptogenesis bound on spontaneous symmetry breaking of global lepton number. Eur. Phys. J. C 71, 1560 (2011). https://doi.org/10.1140/epjc/s10052-011-1560-2. arXiv:0909.5468

106. P.S.B. Dev, R.N. Mohapatra, Y. Zhang, Leptogenesis constraints on $B-L$ breaking Higgs boson in TeV scale seesaw models. JHEP 03, 122 (2018). https://doi.org/10.1007/JHEP03(2018)122. arXiv: 1711.07634

107. E.K. Akhmedov, Z.G. Berezhiani, R.N. Mohapatra, G. Senjanovic, Planck scale effects on the majoron. Phys. Lett. B 299, 90-93 (1993). https://doi.org/10.1016/0370-2693(93)90887-N. arXiv:hep-ph/9209285

108. I.Z. Rothstein, K.S. Babu, D. Seckel, Planck scale symmetry breaking and majoron physics. Nucl. Phys. B 403, 725748 (1993). https://doi.org/10.1016/0550-3213(93)90368-Y. arXiv:hep-ph/9301213

109. A. Hebecker, P. Mangat, S. Theisen, L.T. Witkowski, Can gravitational instantons really constrain axion inflation? JHEP 02, 097 (2017). https://doi.org/10.1007/JHEP02(2017)097. arXiv: 1607.06814

110. R. Alonso, A. Urbano, Wormholes and masses for Goldstone bosons. arXiv: 1706.07415

111. K.-M. Lee, Wormholes and goldstone bosons. Phys. Rev. Lett. 61, 263-266 (1988). https://doi.org/10.1103/PhysRevLett.61.263

112. C.T. Hill, G.G. Ross, Models and new phenomenological implications of a class of pseudogoldstone bosons. Nucl. Phys. B 311, 253-297 (1988). https://doi.org/10.1016/0550-3213(88)90062-4

113. M. Frigerio, T. Hambye, E. Masso, Sub-GeV dark matter as pseudo-Goldstone from the seesaw scale. Phys. Rev. X 1, 021026 (2011). https://doi.org/10.1103/PhysRevX.1.021026. arXiv: 1107.4564

114. M. Lattanzi, J.W.F. Valle, Decaying warm dark matter and neutrino masses. Phys. Rev. Lett. 99, 121301 (2007). https://doi.org/ 10.1103/PhysRevLett.99.121301. arXiv:0705.2406
115. M. Lattanzi, R.A. Lineros, M. Taoso, Connecting neutrino physics with dark matter. New J. Phys. 16(12), 125012 (2014). https://doi. org/10.1088/1367-2630/16/12/125012. arXiv:1406.0004

116. K. Choi, A. Santamaria, Majorons and supernova cooling. Phys. Rev. D 42, 293-306 (1990). https://doi.org/10.1103/PhysRevD. 42.293

117. A. Kusenko, Sterile neutrinos, dark matter, and the pulsar velocities in models with a Higgs singlet. Phys. Rev. Lett. 97, 241301 (2006). https://doi.org/10.1103/PhysRevLett.97.241301. arXiv:hep-ph/0609081

118. K. Petraki, A. Kusenko, Dark-matter sterile neutrinos in models with a gauge singlet in the Higgs sector. Phys. Rev. D 77, 065014 (2008). https://doi.org/10.1103/PhysRevD.77.065014. arXiv:0711.4646

119. N. Sekiya, N. Y. Yamasaki, K. Mitsuda, A Search for a keV signature of radiatively decaying dark matter with Suzaku XIS Observations of the X-ray Diffuse Background, Publ. Astron. Soc. Jap. https://doi.org/10.1093/pasj/psv081. arXiv:1504.02826

120. A. Boyarsky, D. Malyshev, A. Neronov, O. Ruchayskiy, Constraining DM properties with SPI. Mon. Not. R. Astron. Soc. 387, 1345 (2008). https://doi.org/10.1111/j.1365-2966.2008.13003.x. arXiv:0710.4922 